\title{
Asymptotic cones of HNN extensions and amalgamated products
}

\author{
CURTIS KENT
}

\begin{abstract}
Gromov asked whether an asymptotic cone of a finitely generated group was always simply connected or had uncountable fundamental group. We prove that Gromov's dichotomy holds for asymptotic cones with cut points, as well as HNN extensions and amalgamated products where the associated subgroups are nicely embedded. We also show a slightly weaker dichotomy for multiple HNN extensions of free groups.
\end{abstract}

20F65, 20F69; 57M07

\section{Introduction}

Gromov was first to notice a connection between the homotopic properties of asymptotic cones of a finitely generated group and algorithmic properties of the group (Gromov [16, Section 5.F]). Gromov asked what kind of fundamental groups can asymptotic cones of finitely generated groups have [16]. In particular, he asked whether the following dichotomy is true: The fundamental group of an asymptotic cone of a finitely generated group is always either trivial or of order continuum. One reason for this question was that asymptotic cones of nilpotent groups are simply connected (Pansu [28]); the same is true for hyperbolic groups since all cones in that case are $\mathbb{R}$-trees, but asymptotic cones of many solvable non-nilpotent groups (say, the Baumslag-Solitar group BS $(2,1)$ or Sol) contain Hawaiian earrings, and that seems to be a common property of very many other groups (Burillo [3], Conner and Kent [7]).

Answering Gromov's question about possible fundamental groups of asymptotic cones, Erschler and Osin showed that every countable group is a subgroup of the fundamental group of an asymptotic cone of a finitely generated group [13]. Druţu and Sapir proved that, moreover, for every countable group $C$, there exists an asymptotic cone of a finitely generated group $G$ whose fundamental group is the free product of uncountably many copies of $C$ [11]. (Note that for finitely presented groups $G$, analogues of the results of Erschler, Osin, Druţu and Sapir, are still unknown.)

It turned out that Gromov's dichotomy is false: there exists an asymptotic cone of a finitely generated group whose fundamental group is $\mathbb{Z}$ because the cone is homeomorphic to the direct product of a tree and a circle (Ol'shanskii, Osin and Sapir [23]). 
Cornulier and Tessera have produced additional counterexamples by showing that solvable groups can have asymptotic cones with finite fundamental groups [8].

A prairie group is a group where every asymptotic cone of the group is simply connected. A group is constricted if all of its asymptotic cones have (global) cut-points and wide if none of its asymptotic cones have cut-points. We show that for constricted groups Gromov's dichotomy does hold and that a modified version of Gromov's dichotomy holds for groups that are not wide.

Theorem A (Corollary 2.23) Let $G$ be a finitely generated group. If $G$ is constricted, then the fundamental group of an asymptotic cone of $G$ is either trivial or contains an uncountably generated free subgroup. If $G$ is not wide, then $G$ has an asymptotic cone that is simply connected or contains an uncountably generated free subgroup.

If an asymptotic cone of a group contains a cut-point, then it is tree-graded with respect to subsets called pieces that are maximal subsets not containing cut-points. For a definition of tree-graded, see [11]. The naive way to try prove the above theorem is to say that if the fundamental group is non-trivial then some piece contains an essential loop. However, the one-point wedge of two contractible spaces can have uncountable fundamental group (see the remark following Proposition 3.9). Hence, it is possible that all pieces have trivial fundamental group while the asymptotic cone itself has non-trivial fundamental group.

Instead, we show that any ultralimit of compact subsets of an asymptotic cone embeds into the cone (see Proposition 2.19). A slightly stronger version of this statement is proved, assuming the continuum hypothesis, in Osin and Sapir [27]. It is also related to work of Sisto in [32]. Proposition 2.19 provides a way to prove that every maximal transversal tree in the asymptotic cone of a noncyclic group is a universal $\mathbb{R}$-tree. (A tree $T \subset X$ is transversal in $X$ if the connected components of $T \backslash\{t\}$ are contained in distinct connected components of $X \backslash\{t\}$ for every $t \in T$.) This implies the existence of an uncountable set of points such that any two are separated by a cut-point. We then use homogeneity together with this uncountable set of points to prove that any essential loop has uncountably many translates such that any two are separated by a cut-point. These translates serve as generators for a subgroup of the fundamental group that is an uncountable product of cyclic groups.

In the process, we also obtained the following result which is interesting in its own right.

Proposition B (Proposition 2.12) Let $X$ be a homogeneous geodesic metric space. Every asymptotic cone of $X$ is one-ended if and only if $X$ is wide if and only if no asymptotic cone of $X$ has a local cut-point. 
By extending these methods to unbounded sets that separate, we were able to show that Gromov's dichotomy holds for HNN extensions and amalgamated products with nicely embedded associated subgroups.

Theorem C (Theorem 3.16) Suppose that $G$ is an HNN extension or amalgamated product where the associated subgroups are proper, quasi-isometrically embedded, prairie groups. Then every asymptotic cone of $G$ is either simply connected or has uncountable fundamental group.

Another weaker version of Gromov's dichotomy holds for multiple HNN extensions of free groups:

Theorem D (Theorem 4.16) If $G$ is a multiple HNN extension of a free group, then every asymptotic cone of $G$ is simply connected or $G$ has an asymptotic cone with uncountable fundamental group.

Multiple HNN extensions of free groups can have unusual asymptotic properties. Using multiple HNN extensions, Sapir, Birget and Rips were able to construct a finitely presented group with undecidable word problem and cubic Dehn function. They also constructed examples of groups with Dehn function equivalent to $n^{\alpha}$ for any $\alpha \geq 4$ that is computable in time less than $2^{2^{C m}}$ for some constant $C>0$ [31]. Ol'shanskii and Sapir constructed a multiple HNN extension of a free group that has $\pi_{1}$-nonequivalent asymptotic cones [26] and another that has all cones with uncountable fundamental group and $n^{2} \log (n)$ Dehn function [24] (see [7] for a proof that the cones have uncountable $\pi_{1}$ ). As noted earlier, Burillo in [3] showed that Baumslag-Solitar groups can have $\pi_{1}$-embedded Hawaiian earring groups.

When Gromov's dichotomy was formulated, examples of groups with several nonhomeomorphic (or moreover $\pi_{1}$-non-equivalent) asymptotic cones were not known. Now we know that a finitely generated group can have uncountably many pairwise $\pi_{1}$-non-equivalent asymptotic cones [11] (or much more pairwise non-homeomorphic cones, if the continuum hypothesis is assumed false (Kramer, Shelah, Tent and Thomas [19])), so our weaker version of the dichotomy seems natural.

\subsection{Definitions}

Let $G=\langle S\rangle$ be a group and $u, v$ be two words in the alphabet $S$. We write $u \equiv v$ when $u$ and $v$ coincide letter by letter and $u={ }_{G} v$ if $u$ and $v$ are equal in $G$. We will denote the Cayley graph of $G$ with respect to the generating set $S$ by $\Gamma(G, S)$. We will use the standard convention of considering $G$ to be the set of vertices of the Cayley 
graph, which acts on $\Gamma(G, S)$ by isometries. The Cayley complex of $G$ depends on a set of generators and a set of relations but will be denoted $\Gamma^{2}(G, S)$ when the relators are understood. We will use Lab to represent the function from the set of edge paths in a labeled oriented CW-complex to the set of words in the alphabet obtained by reading the label of a path.

We will use $\theta$ to denote the canonical map taking a van Kampen diagram into the Cayley complex that restricts to a label-preserving map on the 1-skeleton of the diagram. Explicitly, let $\Delta$ be a van Kampen diagram with a distinguished vertex $o$, and $g_{o}$ a vertex of $\Gamma(G, S)$. For $v$ a vertex of $\Delta$, let $\theta(v)=g_{0} w_{v}$ where $w_{v}$ is the label of any path in $\Delta$ from $o$ to $v$. Whenever $\Delta$ is a simply connected diagram, this map is independent of the choice of $w_{v}$ and extends to a map on all of $\Delta$ as follows. For $e$ an edge of $\Delta$ labeled by $s$ with initial vertex $v$, let $\theta(e)=(\theta(v), s)$ where $(\theta(v), s)$ is the edge in $\Gamma(G, S)$ with initial vertex $\theta(v)$ and labeled by $s$. For $\pi$ a 2 -cell of $\Delta$, we may choose a vertex $v$ on $\partial \pi$ such that $\mathbf{L a b}(\pi) \equiv r^{ \pm 1}$ with this choice of base point. Then $\theta(\pi)=D_{\theta(v), r^{ \pm 1}}$ where $D_{\theta(v), r^{ \pm 1}}$ is the $2-$ cell in $\Gamma^{2}(G, S)$ with boundary, read from $\theta(v)$, labeled by $r^{ \pm 1}$. The map $\theta$ is unique up to our choice of $g_{0}$ and $o$.

Definition 1.1 Suppose that $\beta$ is a simple closed curve contained in the interior of a planar disc $D$. Then $D \backslash \beta$ has exactly two components. The component of $D \backslash \beta$ whose closure contains $\partial D$ will be called the unbounded component of $D \backslash \beta$. The other component will be called the bounded component. A point $v \in D$ is interior (or exterior) to $\beta$ if it is contained in the bounded (or unbounded) component of $D \backslash \beta$.

Definition 1.2 (Asymptotic cones) Let $\omega$ be an ultrafilter on $\mathbb{N}$ and $c_{n}$ be a sequence of positive real numbers. The sequence $c_{n}$ is bounded $\omega$-almost surely or $\omega$-bounded, if there exists a number $M$ such that $\omega\left(\left\{n \mid c_{n}<M\right\}\right)=1$. If $c_{n}$ is $\omega$-bounded, then there exists a unique number, which we will denote by $\lim ^{\omega} c_{n}$, such that $\omega\left(\left\{n|| c_{n}-\lim ^{\omega} c_{n} \mid<\epsilon\right\}\right)=1$ for every $\epsilon>0$.

If $c_{n}$ is not $\omega$-bounded, then $\omega\left(\left\{n \mid c_{n}>M\right\}\right)=1$ for every $M$. We will say that $c_{n}$ diverges $\omega$-almost surely or is $\omega$-divergent and let $\lim ^{\omega} c_{n}=\infty$.

Let $\left(X_{n}\right.$, dist $\left._{n}\right)$ be a sequence of metric spaces and $\omega$ an ultrafilter on $\mathbb{N}$. Consider a sequence of points $e=\left(e_{n}\right)$ such that $e_{n} \in X_{n}$ called an observation sequence.

Given two elements $x=\left(x_{n}\right), y=\left(y_{n}\right) \in \prod X_{n}$, set $\operatorname{dist}(x, y)=\lim ^{\omega} \operatorname{dist}_{n}\left(x_{n}, y_{n}\right)$. We can then define an equivalence relation $\sim$ on $\prod X_{n}$ by $x \sim y$ if and only if $\operatorname{dist}(x, y)=0$. 
The ultralimit of $X_{n}$ relative to the observation sequence $e$ is:

$$
\lim _{e}^{\omega} X_{n}=\left\{x=\left(x_{n}\right) \in \prod X_{n} \mid \operatorname{dist}(x, e)<\infty\right\} / \sim
$$

Now consider an $\omega$-divergent sequence of numbers $d=\left(d_{n}\right)$ called a scaling sequence and a metric space $(X$, dist).

The asymptotic cone of $X$ with respect to $e, d$ and $\omega$ is

$$
\operatorname{Con}^{\omega}(X, e, d)=\lim _{e}^{\omega}\left(X, \operatorname{dist} / d_{n}\right),
$$

where dist $/ d_{n}$ is the metric on $X$ scaled by $\frac{1}{d_{n}}$.

$\operatorname{Con}^{\omega}(X, e, d)$ is a complete metric space. If $X$ is geodesic, then $\operatorname{Con}^{\omega}(X, e, d)$ is also geodesic. If $X$ is a homogeneous metric space, then the isometry type of $\operatorname{Con}^{\omega}(X, e, d)$ is independent of $e$ and will frequently be denoted by $\operatorname{Con}^{\omega}(X, d)$.

Suppose that $\left\{X_{n}\right\}$ is a sequence of subsets of a metric space (X, dist). At times it will be convenient to talk about the subset of $\operatorname{Con}^{\omega}(X, e, d)$ with representatives in $\prod X_{n}$. When it is clear from the text, we will denote this subset by $\lim ^{\omega} X_{n}$ instead of the more precise $\lim _{e}^{\omega}\left(X_{n}\right.$, dist $\left./ d_{n}\right)$. When used in this context, we will not require that $e_{n}$ be an element of $X_{n}$.

\section{Wide groups and ends of asymptotic cones}

The following lemma is obvious.

Lemma 2.1 Let $\omega$ be an ultrafilter on $\mathbb{N}$ and $d=\left(d_{n}\right)$ a scaling sequence. Suppose that $\left\{\gamma_{n}\right\}$ is a sequence of loops parametrized by arc length in a geodesic metric space ( $X$, dist) such that $\left|\gamma_{n}\right|=O\left(d_{n}\right)$. Then $\gamma(t)=\left(\gamma_{n}(t)\right)$ is a continuous map of $S^{1}$ into $\operatorname{Con}^{\omega}(X, e, d)$.

The converse also holds.

Proposition 2.2 Let $X$ be a geodesic metric space. For every path $\gamma \in \operatorname{Con}^{\omega}(X, e, d)$, there exist paths $\gamma_{n}$ in $X$ such that $\gamma(t)=\left(\gamma_{n}(t)\right)$.

Recall that there exist geodesics in a cone that are not limits of geodesics. However, here we do not put any restraints on the paths $\gamma_{n}$ (the proof shows that $\gamma_{n}$ can be chosen to be a $2^{m_{n}}$-gon, where $m_{n}$ is an $\omega$-divergent sequence). 
Proof Suppose that $\gamma:[0,1] \rightarrow \operatorname{Con}^{\omega}(X, e, d)$ is a path and let $\xi$ be a modulus of continuity for $\gamma$ (see Definition 3.6).

For each diadic rational $r$ in $[0,1]$, fix a representative $\left(a_{n}(r)\right)$ for $\gamma(r)$. Let

$$
\begin{aligned}
& A_{i}=\left\{0, \frac{1}{2^{i}}, \ldots, \frac{2^{i}-1}{2^{i}}, 1\right\}, \\
& C_{i}=\left\{n|| \operatorname{dist}(\gamma(r), \gamma(s))-\frac{\operatorname{dist}\left(a_{n}(r), a_{n}(s)\right)}{d_{n}} \mid \leq \frac{1}{i} \quad \text { for } s, r \in A_{i}\right\} .
\end{aligned}
$$

Then $C_{i}$ is $\omega$-large, since $\left|A_{i}\right|$ is finite and $\left(a_{n}(r)\right)$ is a representative of $\gamma(r)$. Also, $C_{i} \subset C_{i-1} \subset \cdots \subset C_{1}$ is nested.

Let $D_{n}=\left\{i \mid n \in C_{i}\right.$ and $\left.i \leq n\right\}$ and $m_{n}=\max D_{n}$, if $D_{n}$ is non-empty and $m_{n}=1$ otherwise.

\section{Claim $1 \lim ^{\omega} m_{n}=\infty$}

Proof of Claim 1 Suppose that $m_{n}$ was bounded by $L$ on some $\omega$-large set $C$. Fix $n_{0} \in C \cap\{2 L, 2 L+1,2 L+2, \ldots\} \cap C_{2 L}$ (the intersection is non-empty since all three are $\omega$-large). Then $n_{0} \in C_{2 L}$ and $2 L \leq n_{0}$. This implies that $2 L \in D_{n_{0}}$. Hence $m_{n_{0}} \geq 2 L$, which contradicts our assumption that $m_{n}$ was bounded by $L$ on $C$ since $n_{0} \in C$.

By the above argument, $\omega\left(\left\{n \mid m_{n} \leq L\right\}\right)=0$. Hence $\omega\left(\left\{n \mid m_{n}>L\right\}\right)=1$. Since this holds for every $L$, the claim is proved.

Define $\gamma_{n}:[0,1] \rightarrow X$ by

$$
\gamma_{n}(r)=a_{n}(r), \quad \text { for } r \in A_{m_{n}},
$$

and extend $\gamma_{n}$ geodesically.

We can then define $\gamma^{\prime}(t)=\left(\gamma_{n}(t)\right)$.

Let $B_{k}=\left\{n \mid m_{n}>k\right\}$, which is $\omega$-large by the Claim 1. Fix $t_{0}$ in the diadic rationals and $n_{0}$ such that $t_{0} \in A_{n_{0}}$. Then for all $n \in B_{n_{0}}, t_{0} \in A_{m_{n}}$. This implies that $\gamma_{n}\left(t_{0}\right)=$ $a_{n}\left(t_{0}\right)$ for $n \in B_{n_{0}}$. Since $B_{n_{0}}$ is $\omega$-large, $\gamma^{\prime}\left(t_{0}\right)=\left(\gamma_{n}\left(t_{0}\right)\right)=\left(a_{n}\left(t_{0}\right)\right)=\gamma\left(t_{0}\right)$. Hence $\gamma^{\prime}(t)=\gamma(t)$ on the diadic rationales.

Notice by our choice of $m_{n}$, we have $n \in C_{m_{n}}$ if $m_{n} \neq 1$. Thus for $n \in B_{1}$ and $r, s \in A_{m_{n}}$, we have

$$
\operatorname{dist}(\gamma(r), \gamma(s))-\frac{1}{m_{n}} \leq \frac{\operatorname{dist}\left(\gamma_{n}(r), \gamma_{n}(s)\right)}{d_{n}} \leq \operatorname{dist}(\gamma(r), \gamma(s))+\frac{1}{m_{n}} .
$$


Fix $x, y \in[0,1]$. Choose $r_{x}^{1}, r_{x}^{2}, r_{y}^{1}, r_{y}^{2} \in A_{m_{n}}$ such that $x \in\left[r_{x}^{1}, r_{x}^{2}\right], y \in\left[r_{y}^{1}, r_{y}^{2}\right]$ and

$$
d\left(r_{x}^{1}, r_{x}^{2}\right)=d\left(r_{y}^{1}, r_{y}^{2}\right)=\frac{1}{2^{m_{n}}} .
$$

Then for $n \in B_{1},\left.\gamma_{n}\right|_{\left[r_{x}^{1}, r_{x}^{2}\right]},\left.\gamma_{n}\right|_{\left[r_{y}^{1}, r_{y}^{2}\right]}$ are geodesics of length at most

$$
d_{n}\left[\xi\left(\frac{1}{2^{m_{n}}}\right)+\frac{1}{m_{n}}\right]
$$

Thus

$$
\operatorname{dist}\left(\gamma_{n}(x), \gamma_{n}\left(r_{x}^{1}\right)\right), \operatorname{dist}\left(\gamma_{n}\left(r_{y}^{1}\right), \gamma_{n}(y)\right) \leq d_{n}\left[\xi\left(\frac{1}{2^{m_{n}}}\right)+\frac{1}{m_{n}}\right] .
$$

Then combining this with the triangle inequality, we obtain $\operatorname{dist}\left(\gamma_{n}(x), \gamma_{n}(y)\right)$

$$
\begin{aligned}
& \leq \operatorname{dist}\left(\gamma_{n}(x), \gamma_{n}\left(r_{x}^{1}\right)\right)+\operatorname{dist}\left(\gamma_{n}\left(r_{x}^{1}\right), \gamma_{n}\left(r_{y}^{1}\right)\right)+\operatorname{dist}\left(\gamma_{n}\left(r_{y}^{1}\right), \gamma_{n}(y)\right) \\
& \leq d_{n}\left[\xi\left(\frac{1}{2^{m_{n}}}\right)+\frac{1}{m_{n}}\right]+d_{n}\left[\xi\left(\operatorname{dist}\left(r_{x}^{1}, r_{y}^{1}\right)\right)+\frac{1}{m_{n}}\right]+d_{n}\left[\xi\left(\frac{1}{2^{m_{n}}}\right)+\frac{1}{m_{n}}\right] \\
& =2 d_{n} \xi\left(\frac{1}{2^{m_{n}}}\right)+\frac{3 d_{n}}{m_{n}}+d_{n} \xi\left(\operatorname{dist}(x, y)+\frac{2}{2^{m_{n}}}\right)
\end{aligned}
$$

Then

$$
\begin{aligned}
\operatorname{dist}\left(\gamma^{\prime}(x), \gamma^{\prime}(y)\right) & \leq \lim _{n} \omega \frac{\operatorname{dist}\left(\gamma_{n}(x), \gamma_{n}(y)\right)}{d_{n}} \\
& \leq \lim _{n} \omega 2 \xi\left(\frac{1}{2^{m_{n}}}\right)+\frac{3}{m_{n}}+\xi\left(d(x, y)+\frac{2}{2^{m_{n}}}\right)=\xi(d(x, y)),
\end{aligned}
$$

which implies that $\gamma^{\prime}$ is continuous. (Note that we used that fact that $\xi$ was a continuous modulus of continuity.) Hence $\gamma(t)=\gamma^{\prime}(t)$ for all $t$.

Proposition 2.3 Let $X$ be a simply connected geodesic metric space that has a linear isodiametric function. Suppose that $h: \mathbb{D} \rightarrow \operatorname{Con}^{\omega}(X, e, d)$ is a continuous map of the unit disc. Then there exist continuous maps $h_{n}: \mathbb{D} \rightarrow X$ such that $\gamma(t)=\left(\gamma_{n}(t)\right)$.

The proof is very similar to that of Proposition 2.2. However, we present it here to illustrate how to modify the proof of Proposition 2.2 for discs of higher dimension.

Proof For simplicity of notation, we will assume $\mathbb{D}=[0,1]^{2}$. Fix $M$ such that every loop of length at most $n$ bounds a disc of diameter at most $M n$.

Suppose that $h:[0,1]^{2} \rightarrow \operatorname{Con}^{\omega}(X, e, d)$ is a continuous map and let $\xi$ be a modulus of continuity for $h$. 
For each diadic rational pair $\vec{r}=(r, s)$, fix a representative $\left(a_{n}(\vec{r})\right)$ of $h(\vec{r})$. Let

$$
A_{i}=\left\{\vec{r}=(r, s) \mid r, s \in\left\{0, \frac{1}{2^{i}}, \ldots, \frac{2^{i}-1}{2^{i}}, 1\right\}\right\} .
$$

We can consider $A_{i}$ as the vertices of a cellular decomposition of $\mathbb{D}$ into squares with side length $\frac{1}{2^{i}}$ and denote the $j$-skeleton of this decomposition by $A_{i}^{(j)}$ for $j=1,2$.

$$
C_{i}=\left\{n|| \operatorname{dist}(h(\vec{r}), h(\vec{s}))-\frac{\operatorname{dist}\left(a_{n}(\vec{r}), a_{n}(\vec{s})\right)}{d_{n}} \mid \leq \frac{1}{i} \text { for } \vec{r}, \vec{s} \in A_{i}\right\} .
$$

Then $C_{i}$ is $\omega$-large, since $\left|A_{i}\right|$ is finite and $\left(a_{n}(\vec{r})\right)$ is a representative of $h(\vec{r})$. Also, $C_{i} \subset C_{i-1} \subset \cdots \subset C_{1}$ is nested.

Let $D_{n}=\left\{i \mid n \in C_{i}\right.$ and $\left.i \leq n\right\}$ and $m_{n}=\max D_{n}$, if $D_{n}$ is non-empty and $m_{n}=1$ otherwise.

\section{Claim $2 \lim ^{\omega} m_{n}=\infty$}

Proof of Claim 2 The proof is identical to that of Claim 1 from the previous proposition.

Define $h_{n}: A_{m_{n}}^{(1)} \rightarrow X$ by

$$
h_{n}(\vec{r})=a_{n}(\vec{r}), \quad \text { for } \vec{r} \in A_{m_{n}},
$$

and extend $f_{n}$ geodesically to all of $A_{m_{n}}^{(1)}$. Let $e$ be a 2-cell of $A_{m_{n}}$. Then $\left|h_{n}(\partial e)\right|$ is at most $4 \operatorname{diam}\left(h_{n}(\partial e)\right)$ and we can extend $h_{n}$ to $\mathbb{D}$ by mapping each 2-cell $e$ of $A_{m_{n}}$ to a disc of diameter at most $4 M \operatorname{diam}\left(h_{n}(\partial e)\right)$.

We can now define $h^{\prime}: \mathbb{D} \rightarrow \operatorname{Con}^{\omega}(X, e, d)$ by $h^{\prime}(\vec{t})=\left(h_{n}(\vec{t})\right)$ for all $\vec{t} \in \mathbb{D}$.

Let $B_{k}=\left\{n \mid m_{n}>k\right\}$, which is $\omega$-large by the claim. Fix a diadic rational pair $\vec{r}_{0}=\left(r_{0}, s_{0}\right)$ and $n_{0}$ such that $\vec{r}_{0} \in A_{n_{0}}$. Then for all $n \in B_{n_{0}}, \vec{r}_{0} \in A_{m_{n}}$. This implies that $h_{n}\left(\vec{r}_{0}\right)=a_{n}\left(\vec{r}_{0}\right)$ for $n \in B_{n_{0}}$. Since $B_{n_{0}}$ is $\omega$-large, $h^{\prime}\left(\vec{r}_{0}\right)=\left(h_{n}\left(\vec{r}_{0}\right)\right)=$ $\left(a_{n}\left(\vec{r}_{0}\right)\right)=h\left(\vec{r}_{0}\right)$. Hence $h^{\prime}(\vec{r})=h(\vec{r})$ for any diadic pair $\vec{r}=(r, s)$.

Notice by our choice of $m_{n}$, we have $n \in C_{m_{n}}$ if $m_{n} \neq 1$. Thus for $n \in B_{1}$ and $\vec{r}, \vec{s} \in A_{m_{n}}$, we have

$$
\operatorname{dist}(h(\vec{r}), h(\vec{s}))-\frac{1}{m_{n}} \leq \frac{\operatorname{dist}\left(h_{n}(\vec{r}), h_{n}(\vec{s})\right)}{d_{n}} \leq \operatorname{dist}(h(\vec{r}), h(\vec{s}))+\frac{1}{m_{n}} .
$$

Fix $\vec{x}_{1}, \vec{x}_{2} \in \mathbb{D}$. Choose $2-$ cells $e_{1}, e_{2}$ of $A_{m_{n}}$ such that $\vec{x}_{i} \in e_{i}$ for $i=1,2$. Also, choose a vertex $\vec{r}_{i}$ of $e_{i}$ for $i=1,2$. 
Then for $n \in B_{1}, \operatorname{diam}\left(h_{n}\left(e_{i}\right)\right) \leq 4 M \operatorname{diam}\left(h_{n}\left(\partial e_{i}\right)\right)$. Since $h_{n}\left(\partial e_{i}\right)$ is a geodesic 4gon, $\operatorname{diam}\left(h_{n}\left(\partial e_{i}\right)\right)$ is at most twice the maximum distance between adjacent vertices. This with Equation (1) implies that

$$
\operatorname{diam}\left(h_{n}\left(e_{i}\right)\right) \leq d_{n} M 8\left[\xi\left(\frac{1}{2^{m_{n}}}\right)+\frac{1}{m_{n}}\right] .
$$

Thus for $i=1,2$ we have

$$
\operatorname{dist}\left(h_{n}\left(\vec{x}_{i}\right), h_{n}\left(\vec{r}_{i}\right)\right) \leq d_{n} M 8\left[\xi\left(\frac{1}{2^{m_{n}}}\right)+\frac{1}{m_{n}}\right] .
$$

Then combining this with the triangle inequality, we obtain:

$$
\begin{aligned}
& \operatorname{dist}\left(h_{n}\left(\vec{x}_{1}\right), h_{n}\left(\vec{x}_{2}\right)\right) \\
& \quad \leq \operatorname{dist}\left(h_{n}\left(\vec{x}_{1}\right), h_{n}\left(\vec{r}_{1}\right)\right)+\operatorname{dist}\left(h_{n}\left(\vec{r}_{1}\right), h_{n}\left(\vec{r}_{2}\right)\right)+\operatorname{dist}\left(h_{n}\left(\vec{r}_{2}\right), h_{n}\left(\vec{x}_{2}\right)\right) \\
& \quad \leq d_{n} M 8\left[\xi\left(\frac{1}{2^{m_{n}}}\right)+\frac{1}{m_{n}}\right]+d_{n}\left[\xi\left(\operatorname{dist}\left(\vec{r}_{1}, \vec{r}_{2}\right)\right)+\frac{1}{m_{n}}\right]+d_{n} M 8\left[\xi\left(\frac{1}{2^{m_{n}}}\right)+\frac{1}{m_{n}}\right] \\
& \quad=16 d_{n} M \xi\left(\frac{1}{2^{m_{n}}}\right)+\frac{d_{n}(16 M+1)}{m_{n}}+d_{n} \xi\left(\operatorname{dist}\left(\vec{x}_{1}, \vec{x}_{2}\right)+\frac{2}{2^{m_{n}}}\right)
\end{aligned}
$$

Then

$$
\begin{aligned}
\operatorname{dist}\left(h^{\prime}\left(\vec{x}_{1}\right), h^{\prime}\left(\vec{x}_{2}\right)\right) & \leq \lim _{n} \omega \frac{\operatorname{dist}\left(h_{n}\left(x_{1}\right), h_{n}\left(\vec{x}_{2}\right)\right)}{d_{n}} \\
& \leq \lim _{n} \omega 16 M \xi\left(\frac{1}{2^{m_{n}}}\right)+\frac{(16 M+1)}{m_{n}}+\xi\left(\operatorname{dist}\left(\vec{x}_{1}, \vec{x}_{2}\right)+\frac{2}{2^{m_{n}}}\right) \\
& =\xi\left(d\left(\vec{x}_{1}, \vec{x}_{2}\right)\right)
\end{aligned}
$$

which implies that $h^{\prime}$ is continuous. (Note that we used that fact that $\xi$ was a continuous modulus of continuity.) Hence $h(\vec{x})=h^{\prime}(\vec{x})$ for all $\vec{x} \in \mathbb{D}$.

Throughout this paper, we will assume that metric balls are open. When $\tau$ is a path in a metric space, we will use $|\tau|$ to denote its arc length. Then $|\cdot|$ maps the set of paths into the extended real line and is finite for rectifiable paths and $+\infty$ for non-rectifiable paths. We will assume that rectifiable paths are parametrized proportional to arc length. We will use $\mathcal{N}_{S}(B)$ to represent the $s$-neighborhood of $B$.

Lemma 2.4 Let $X$ be a homogeneous geodesic metric space. The non-empty components $\operatorname{Con}^{\omega}(X, e, d) \backslash\left\{\left(x_{n}\right)\right\}$ are unbounded for all $\left(x_{n}\right) \in \operatorname{Con}^{\omega}(X, e, d)$.

Proof The lemma is trivial if $X$ is bounded.

Claim Every asymptotic cone of an unbounded homogeneous geodesic metric space contains a bi-infinite geodesic. 
Proof of claim Since $X$ is unbounded and geodesic, there exists a geodesic $\gamma_{n}:\left[-n d_{n}, n d_{n}\right] \rightarrow X$ every $n$. By homogeneity, we may assume that $\gamma_{n}(0)=e_{n}$. We can extend $\gamma_{n}$ to all of $\mathbb{R}$ by $\gamma_{n}(t)=\gamma_{n}\left(n d_{n}\right)$ for $t \geq n d_{n}$ and $\gamma_{n}(t)=\gamma_{n}\left(-n d_{n}\right)$ for $t \leq-n d_{n}$. Define $\gamma: \mathbb{R} \rightarrow \operatorname{Con}^{\omega}(X, e, d)$ by $\gamma(t)=\left(\gamma_{n}\left(t d_{n}\right)\right)$. Then

$$
\operatorname{dist}(\gamma(s), \gamma(t))=\lim ^{\omega} \frac{\operatorname{dist}_{n}\left(\gamma_{n}\left(s d_{n}\right), \gamma_{n}\left(s d_{n}\right)\right)}{d_{n}}=\lim ^{\omega} \frac{\left|s d_{n}-t d_{n}\right|}{d_{n}}=|s-t|,
$$

which completes the proof.

Since $\operatorname{Con}^{\omega}(X, e, d)$ is also homogeneous, it contains a bi-infinite geodesic through every point. Suppose that $A$ is a non-empty connected component of $\operatorname{Con}^{\omega}(X, e, d) \backslash\{x\}$ for some $x \in \operatorname{Con}^{\omega}(X, e, d)$. Let $a \in A$. Then there exists a bi-infinite geodesic $\alpha: \mathbb{R} \rightarrow \operatorname{Con}^{\omega}(X, e, d)$ such $\alpha(0)=a$. Only one of $\alpha((-\infty, 0]), \alpha([0, \infty))$ can intersect $x$. Hence $A$ must contain an unbounded ray.

This lemma also follows from Druţu, Mozes and Sapir [10, Lemma 3.12].

Lemma 2.5 Let $B_{n}$ be a sequence of uniformly bounded subsets of a geodesic metric space $X$ and $\kappa: \mathbb{N} \rightarrow \mathbb{R}$ be a sublinear function. If $X \backslash \mathcal{N}_{k\left(d_{n}\right)}\left(B_{n}\right)$ has more than one unbounded connected component, then, for $e_{n} \in B_{n}, \operatorname{Con}^{\omega}(X, e, d) \backslash \lim _{e}^{\omega} B_{n}$ has more than one unbounded connected component.

Proof Let $\left\{U_{n, 1}, \ldots, U_{n, i_{n}}\right\}$ be the set of unbounded connected components of $X \backslash \mathcal{N}_{k\left(d_{n}\right)}\left(B_{n}\right)$. Let $B=\lim _{e}^{\omega} B_{n}, Z=\lim _{e}^{\omega} U_{n, s_{n}}$ and $Y=\lim _{e}^{\omega} U_{n, t_{n}}$, where $s_{n}, t_{n}$ are distinct elements of $\left\{1, \ldots, i_{n}\right\} \omega$-almost surely. Since $B_{n}$ is uniformly bounded and $U_{n, i}$ is unbounded, both $Y \backslash B$ and $Z \backslash B$ are nonempty and hence unbounded.

Suppose that $x \in Z \cap Y$. Then $x=\left(z_{n}\right)=\left(y_{n}\right)$, where $z_{n} \in U_{n, s_{n}}$ and $y_{n} \in$ $U_{n, t_{n}}$. Since $U_{n, s_{n}}$ and $U_{n, t_{n}}$ are in distinct connected components of $X \backslash \mathcal{N}_{k\left(d_{n}\right)}\left(B_{n}\right)$, every path originating in $U_{n, s_{n}}$ and terminating in $U_{n, t_{n}}$ passes through $\mathcal{N}_{k\left(d_{n}\right)}\left(B_{n}\right)$. By considering a geodesic from $z_{n}$ to $y_{n}$, we can find $b_{n}^{\prime} \in \mathcal{N}_{k\left(d_{n}\right)}\left(B_{n}\right)$ such that $\operatorname{dist}\left(z_{n}, b_{n}^{\prime}\right)+\operatorname{dist}\left(b_{n}^{\prime}, y_{n}\right)=\operatorname{dist}\left(z_{n}, y_{n}\right)$, which implies that $x=\left(b_{n}^{\prime}\right)$. Also, there exists $b_{n} \in B_{n}$ such that $\operatorname{dist}\left(b_{n}, b_{n}^{\prime}\right) \leq \kappa\left(d_{n}\right)$. Hence $x=\left(b_{n}^{\prime}\right)=\left(b_{n}\right)$.

Thus $Z \cap Y \subset B$ and the components of $Z \backslash B, Y \backslash B$ are distinct unbounded components of $\operatorname{Con}^{\omega}(X, e, d) \backslash B$.

At times it will be convenient to consider separating sets which are unbounded. 
Lemma 2.6 Let $\left(B_{n}, e_{n}\right)$ be a sequence of pointed subsets of a geodesic metric space $X, \omega$ an ultrafilter and $d=\left(d_{n}\right)$ an $\omega$-divergent sequence. Suppose that $a=\left(a_{n}\right), b=\left(b_{n}\right) \in \operatorname{Con}^{\omega}(X, e, d)$ are points such that there exists a sublinear function $\kappa: \mathbb{N} \rightarrow \mathbb{R}$ such that $a_{n}, b_{n}$ are in distinct components of $X \backslash \mathcal{N}_{\kappa\left(d_{n}\right)}\left(B_{n}\right)$ $\omega$-almost surely.

Then $\lim _{e}^{\omega} B_{n}$ separates $\operatorname{Con}^{\omega}(X, e, d)$ into at least two connected components and $a$, $b$ are in distinct components of $\operatorname{Con}^{\omega}(X, e, d) \backslash \lim _{e}^{\omega} B_{n}$.

The proof is the same as for bounded sets; the only difference is that we are not able to conclude that the components are unbounded since we cannot apply Lemma 2.4.

Definition 2.7 Let $X$ be a connected, locally connected topological space. A point $x \in X$ is a local cut-point if there exists an open connected neighborhood $U$ of $x$ such that $U \backslash\{x\}$ has at least two connected components. A point $x \in X$ is a global cut-point if $X \backslash\{x\}$ has at least two connected components. $X$ is wide if none of its asymptotic cones has a global cut-point. $X$ is unconstricted if one of its asymptotic cones has no global cut-points. $X$ is constricted if all of its asymptotic cones have global cut-points.

Let $B_{1} \subset B_{2} \subset \cdots$ be an ascending sequence of bounded sets in a metric space $X$ such that every set of bounded diameter is eventually contained in $B_{n}$ for some $n$. This implies that $\cup_{n} B_{n}=X$.

Two descending sequences $U_{1} \supset U_{2} \supset \cdots$ and $V_{1} \supset V_{2} \supset \cdots$ of subsets of $X$ are equivalent if for every $n$ there exist integers $m, k$ such that $V_{m} \subset U_{n}$ and $U_{k} \subset V_{n}$.

An end of $X$ is a descending sequence $U_{1} \supset U_{2} \supset \cdots$ where $U_{i}$ is an unbounded component of $X \backslash B_{i}$. It can be shown that up to the given equivalence on descending sequences of subsets of $X$ the set of ends of $X$ does not depend on $\left\{B_{n}\right\}$.

A metric space $X$ is one-ended if $X \backslash B$ has a unique unbounded connected component for every bounded subset $B$ of $X$.

We will use the following definition and lemma from [10].

Definition 2.8 Let $X$ be a geodesic metric space, and let $0<\delta<1$ and $\gamma \geq 0$. Let $a, b, c \in X$ with $\operatorname{dist}(c,\{a, b\})=r>0$, where $\operatorname{dist}(c,\{a, b\})$ is the minimum of $\operatorname{dist}(c, a)$ and $\operatorname{dist}(c, b)$. Define $\operatorname{div}_{\gamma}(a, b, c ; \delta)$ as the infimum of the lengths of paths $a, b$ that avoid the ball $\mathrm{B}(c, \delta r-\gamma)$ (note that by definition a ball of non-positive radius is empty). If no such path exists, take $\operatorname{div}_{\gamma}(a, b, c ; \delta)=\infty$. 
Lemma 2.9 [10, Lemma 3.14] Let $X$ be a geodesic metric space. Let $\omega$ be any ultrafilter and $d=\left(d_{n}\right)$ be an $\omega$-divergent sequence. Let $a=\left(a_{n}\right), b=\left(b_{n}\right), c=$ $\left(c_{n}\right) \in \operatorname{Con}^{\omega}(X, e, d)$. Let $r=\operatorname{dist}(c,\{a, b\})$. The following conditions are equivalent for any $0 \leq \delta<1$.

(i) The closed ball $\overline{\mathrm{B}}(c, \delta)$ in $\operatorname{Con}^{\omega}(X, e, d)$ separates $a$ from $b$.

(ii) For every $\delta^{\prime}>\delta$ and every (some) $\gamma \geq 0$,

$$
\lim ^{\omega} \frac{\operatorname{div}_{\gamma}\left(a_{n}, b_{n}, c_{n} ; \frac{\delta^{\prime}}{r}\right)}{d_{n}}=\infty .
$$

The following proposition is immediate, as it holds for all homogeneous geodesic metric spaces; see Geoghegan [14].

Proposition 2.10 An asymptotic cone of a finitely generated group can have 0, 1, 2 or uncountable many ends.

Lemma 2.11 Let $X$ be a homogenous geodesic metric space. If $\operatorname{Con}^{\omega}(X, e, d)$ has a local cut-point, then there exists a cone of $X$ with a global cut-point.

Proof Suppose that $\operatorname{Con}^{\omega}(X, e, d)$ has a local cut-point. By homogeneity, $\tilde{x}=\left(x_{n}\right)$ is a local cut-point. Suppose that $U$ is an open connected neighborhood of $\tilde{x}$ such that $U \backslash\{\tilde{x}\}$ has two components.

Claim There exists an $\epsilon>0$ such that $\tilde{x}$ separates every ball about $\tilde{x}$ with radius at most $\epsilon$.

Let $B_{\epsilon}$ be the ball in $\operatorname{Con}^{\omega}(X, e, d)$ about $\tilde{x}$ of radius $\epsilon$.

Fix $\epsilon>0$ such that $B_{\epsilon}$ is a subset of $U$. Let $u, v$ be elements of $U$ that are in different components of $U \backslash\{\tilde{x}\}$. Any path in $U$ from $u$ to $v$ passes through $\tilde{x}$. (Since $\operatorname{Con}^{\omega}(X, e, d)$ is locally path connected and $U$ is open and connected, $U$ is path connected.) Hence, we can find a path $f:[0,1] \rightarrow U$ such that $f^{-1}(\tilde{x})=\left\{\frac{1}{2}\right\}$ and $f(0), f(1)$ are in different components of $U \backslash\{\tilde{x}\}$. This implies that the inclusion map from $B_{\epsilon^{\prime}} \backslash\{\tilde{x}\}$ to $U \backslash\{\tilde{x}\}$ is not contained in a single component for any $\epsilon^{\prime} \leq \epsilon$. Thus $B_{\epsilon^{\prime}} \backslash\{\tilde{x}\}$ is also not connected for any $\epsilon^{\prime} \leq \epsilon$, which completes the proof of the claim.

We can now consider the cones $X_{k}^{\omega}=\operatorname{Con}^{\omega}\left(X,\left(e_{n}\right),\left(d_{n} / k\right)\right)$. It is easy to see that $\tilde{x}$ is a cut-point of the ball of radius $k \epsilon$ in $X_{k}$. Hence, $\lim ^{\omega} X_{k}$ has a global cut point and by [11, Corollary 3.24] $\lim ^{\omega} X_{k}$ is again an asymptotic cone of $X$. 
Proposition 2.12 Let $X$ be a homogeneous geodesic metric space. Every asymptotic cone of $X$ is one-ended if and only if $X$ is wide if and only if no asymptotic cone of $X$ has a local cut-point.

Proof That $X$ is wide if and only if no asymptotic cone of $X$ has a local cut-point follows immediately from the previous lemma. Thus we need only prove that every asymptotic cone of $X$ is one-ended if and only if $X$ is wide.

The "only if" direction of this equivalence is trivial. We must show that if no asymptotic cone of $X$ has a cut-point, then every asymptotic cone of $X$ is one-ended. Suppose that no asymptotic cone of $X$ has a cut-point but $\operatorname{Con}^{\omega}(X, e, d)$ is not one-ended for some choice of $\omega, e, d$. Hence, there exists a bounded subset $\widetilde{B}$ of $\operatorname{Con}^{\omega}(X, e, d)$ such that $\operatorname{Con}^{\omega}(X, e, d) \backslash \widetilde{B}$ has at least two unbounded components. By homogeneity, we may assume that $\tilde{x}=\left(x_{n}\right) \in \widetilde{B}$.

By Lemma 2.5,

$$
\tilde{Y}=\operatorname{Con}^{\omega}\left(\operatorname{Con}^{\omega}(X, e, d),(\tilde{x}),(n)\right) \backslash \lim ^{\omega} B
$$

has more than one connected component. Since $\widetilde{B}$ is bounded, $\lim ^{\omega} \widetilde{B}$ is a point in $\widetilde{Y}$ that separates. Thus it is a cut-point of $\tilde{Y} . \tilde{Y}$ is again an asymptotic cone of $X[11$, Corollary 3.24]. This contradicts the hypothesis that no cone of $X$ has a cut-point.

In [10, Theorem 1.4], Druţu, Mozes and Sapir show that certain semisimple Lie groups (namely those specified in the theorem below) are wide. Hence, we can apply Proposition 2.12 to obtain the following result.

Theorem 2.13 Let $\Gamma$ be an irreducible lattice in a semisimple Lie group of $\mathbb{R}$-rank at least 2. Suppose that $\Gamma$ is either of $\mathbb{Q}$-rank 1 or is of the form $\operatorname{SL}_{n}\left(\mathcal{O}_{\mathcal{S}}\right)$ where $n \geq 3$, $\mathcal{S}$ is a finite set of valuations of a number field $K$ including all infinite valuations, and $\mathcal{O}_{\mathcal{S}}$ is the corresponding ring of $\mathcal{S}$-integers. Then every asymptotic cone of $\Gamma$ is one-ended.

Proposition 2.12 together with [11, Corollary 6.13] gives us the following.

Theorem 2.14 Let $G$ be a finitely generated non-virtually cyclic group satisfying a law. Then all asymptotic cones of $G$ are one-ended.

The following proposition is well known. We present it here only for comparison with Proposition 2.16. 
Proposition 2.15 Let $G$ be a finitely generated group. The following are equivalent:

(a) $G$ is finite.

(b) $G$ has an asymptotic cone that is a point.

(c) $G$ has an asymptotic cone with 0 ends.

Proof If $G$ is an infinite finitely generated group, then $\Gamma(G, S)$ contains a bi-infinite geodesic for every finite generating set $S$. Thus $\operatorname{Con}^{\omega}(G, d)$ contains a bi-infinite geodesic for every infinite group $G$. If $G$ is finite then $\Gamma(G, S)$ is bounded for every generating set $S$ and $\operatorname{Con}^{\omega}(G, d)$ is a point for every pair $(\omega, d)$. Thus (a) and (b) are equivalent. Clearly, (b) implies (c). If $\operatorname{Con}^{\omega}(G, d)$ has 0 ends for some pair $(\omega, d)$, then it does not contain a bi-infinite geodesic. Hence (c) implies (a).

Proposition 2.16 Let $G$ be a finitely generated group. The following are equivalent:

(a) $G$ is infinite and virtually cyclic.

(b) $G$ has an asymptotic cone which is a line.

(c) $G$ has an asymptotic cone with exactly 2 ends.

Proof If $G$ is infinite and virtually cyclic, then $\operatorname{Con}^{\omega}(G, d)$ is a line for every pair $(\omega, d)$. Thus (a) implies (b). The implication $(b) \Rightarrow(a)$ is [11, Corollary 6.2]; also, it also follows from Point [30], since a line has finite Minkowski dimension.

Thus we need only show that if $\operatorname{Con}^{\omega}(G, d)$ has exactly two ends for some pair $(\omega, d)$, then $G$ has an asymptotic cone which is a line.

Suppose that $\operatorname{Con}^{\omega}(G, d) \backslash B_{\rho}\left(x_{0}\right)$ has exactly two unbounded components for some $\rho>0$ and $x_{0} \in \operatorname{Con}^{\omega}(G, d)$. For each $i$, let $U_{i}$ and $V_{i}$ be the two unbounded components of $\operatorname{Con}^{\omega}(G, d) \backslash B_{i \rho}\left(x_{0}\right)$. We may assume that we have chosen $U_{i}, V_{i}$ such that $U_{i} \supset U_{i+1}$ and $V_{i} \supset V_{i+1}$ for all $i$. Fix $x_{i} \in U_{i}$ and $x_{-i} \in V_{i}$ such that $\operatorname{dist}\left(x_{0}, x_{ \pm i}\right)=i \rho$ for all $i \in \mathbb{N}$.

Define a path $\alpha: \mathbb{R} \rightarrow \operatorname{Con}^{\omega}(G, d)$ by $\alpha(i)=x_{i}$, for $i \in \mathbb{Z}$, and for every $i \in \mathbb{Z}$ extend $\alpha$ to $[i, i+1]$ by sending the interval to a geodesic joining its endpoints.

Claim $3 \alpha$ is a quasi-geodesic with constants depending only on $\rho$, and $\operatorname{Con}^{\omega}(G, d)$ is contained in the $2 \rho$-neighborhood of the image of $\alpha$.

Notice that Claim 3 implies that $G$ has an asymptotic cone that is a line since any asymptotic cone of $\operatorname{Con}^{\omega}(G, d)$ is a line and an asymptotic cone of $G$.

Let $\alpha_{i}^{-}=\alpha((-\infty, i-4]), \alpha_{i}^{+}=\alpha([i+4, \infty))$ and $Y_{i}=\operatorname{Con}^{\omega}(G, d) \backslash B_{\rho}\left(x_{i}\right)$ for all $i$. By homogeneity, $Y_{i}$ has exactly two unbounded connected components. 
Subclaim 3.1 For all $i, j \in \mathbb{Z}$, $\operatorname{dist}\left(x_{i}, x_{j}\right) \geq|j-i| \rho-2 \rho$ and hence $\alpha_{i}^{ \pm} \subset Y_{i}$.

Proof of Subclaim 3.1 If $i, j$ have the same sign then by applying the triangle inequality to a geodesic triangle with vertices $x_{0}, x_{i}, x_{j}$, we obtain $\operatorname{dist}\left(x_{i}, x_{j}\right) \geq$ $|j-i| \rho$.

Suppose that $i \leq 0 \leq j$. By construction, every geodesic from $x_{i}$ to $x_{j}$ passes within $\rho$ of $x_{0}$. Fix a geodesic from $x_{i}$ to $x_{j}$ and let $x_{0}^{\prime}$ be a point on the geodesic such that $\operatorname{dist}\left(x_{0}, x_{0}^{\prime}\right) \leq \rho$. Then $-i \rho=\operatorname{dist}\left(x_{i}, x_{0}\right) \leq \operatorname{dist}\left(x_{i}, x_{0}^{\prime}\right)+\rho$ and $j \rho=\operatorname{dist}\left(x_{0}, x_{j}\right) \leq$ $\operatorname{dist}\left(x_{0}^{\prime}, x_{j}\right)+\rho$, which gives us that $(j-i) \rho \leq \operatorname{dist}\left(x_{i}, x_{0}^{\prime}\right)+\operatorname{dist}\left(x_{0}^{\prime}, x_{j}\right)+2 \rho=$ $\operatorname{dist}\left(x_{i}, x_{j}\right)+2 \rho$. Thus $\operatorname{dist}\left(x_{i}, x_{j}\right) \geq|j-i| \rho-2 \rho$. If $j \in(\infty, i-4] \cup[i+4, \infty)$, then $\operatorname{dist}\left(x_{i}, x_{j}\right) \geq 2 \rho$. Since every point on $\alpha_{i}^{ \pm}$is within $\rho$ of some $x_{j}$ for $j \in$ $(\infty, i-4] \cup[i+4, \infty), \alpha_{i}^{ \pm} \subset Y_{i}$.

Subclaim 3.2 $\alpha_{i}^{+}, \alpha_{i}^{-}$are contained in distinct unbounded components of $Y_{i}$ for all $i$.

Proof of Subclaim 3.2 We will show the subclaim for $i \geq 0$. The other case is similar. Let $U, V$ be the two disjoint unbounded components of $Y_{i}$. By way of contradiction, we will assume that $\alpha_{i}^{ \pm}$are both contained in $U$. Choose $\tilde{g} \in \prod G$ such that $\tilde{g} \cdot x_{0}=x_{i}$. For each $j \geq 1$, let $\beta_{j}^{-}=\alpha((-\infty,-2 j-4]), \beta_{j}^{+}=\alpha([2 j+4, \infty))$. Since $\tilde{g}$ acts by isometries on $\operatorname{Con}^{\omega}(G, d)$, we obtain that $Y_{i}=\tilde{g} \cdot Y_{0}$ and $\tilde{g} \cdot \beta_{j}^{ \pm}$are in distinct unbounded components of $Y_{i}$ for any $j \geq 1$.

Fix $j \geq i$. Since $\alpha_{i}^{-}, \alpha_{i}^{+}$are contained in the same connected component of $Y_{i}$ and $\tilde{g} \cdot \beta_{j}^{-}, \tilde{g} \cdot \beta_{j}^{+}$are contained in distinct connected components of $Y_{i}$, one of $\tilde{g} \cdot \beta_{j}^{ \pm}$is contained in $V$. Suppose that $\tilde{g} \cdot \beta_{j}^{+} \subset V$. (Again the other case is similar.) Notice that $\beta_{j}^{ \pm} \subset \alpha_{i}^{ \pm}$which implies that $\beta_{j}^{-} \cup \beta_{j}^{+} \subset U$.

By Subclaim 3.1, $\operatorname{dist}\left(x_{i}, \tilde{g} \cdot \beta_{j}^{ \pm}\right) \geq(2 j+4) \rho-2 \rho$, which implies that $\operatorname{dist}\left(x_{0}, \tilde{g} \cdot \beta_{j}^{ \pm}\right) \geq$ $(j+2) \rho$. Thus

$$
\tilde{g} \cdot \beta_{j}^{ \pm} \subset \operatorname{Con}^{\omega}(G, d) \backslash B_{(j+1) \rho}\left(x_{0}\right) .
$$

Again by Subclaim 3.1, we have that $\beta_{j}^{ \pm} \subset \operatorname{Con}^{\omega}(G, d) \backslash B_{(j+1) \rho}\left(x_{0}\right)$. Since $B_{\rho}\left(x_{0}\right)$ and $B_{\rho}\left(x_{i}\right)$ are contained in $B_{(j+1) \rho}\left(x_{0}\right)$, each of the three unbounded sets $\beta_{j}^{ \pm}, \widetilde{g} \cdot \beta_{j}^{+}$ must be contained in a distinct connected component of $\operatorname{Con}^{\omega}(G, d) \backslash B_{(j+1) \rho}\left(x_{0}\right)$. Since this holds for any $j \geq i, \operatorname{Con}^{\omega}(G, d)$ must have at least 3 ends, which contradicts our assumption that $\operatorname{Con}^{\omega}(G, d)$ has exactly 2 ends.

Proof of Claim 3 If $i, j \in \mathbb{Z}$ have different signs, then $\operatorname{dist}\left(x_{i}, x_{j}\right) \leq \operatorname{dist}\left(x_{i}, x_{0}\right)+$ $\operatorname{dist}\left(x_{j}, x_{0}\right)=|i| \rho+|j| \rho=|i-j| \rho$. 
For $4 \leq i \leq j-4$, any geodesic from $x_{0}$ to $x_{j}$ is passes within $\rho$ of $x_{i}$ by Subclaim 3.2. Hence, we may find a point $x_{i}^{\prime}$ on a geodesic from $x_{0}$ to $x_{j}$ such that $\operatorname{dist}\left(x_{i}, x_{i}^{\prime}\right) \leq \rho$. Then $i \rho \leq \operatorname{dist}\left(x_{0}, x_{i}^{\prime}\right)+\rho$ and $\operatorname{dist}\left(x_{i}, x_{j}\right) \leq \operatorname{dist}\left(x_{i}^{\prime}, x_{j}\right)+\rho$, which implies that $\operatorname{dist}\left(x_{i}, x_{j}\right) \leq(j-i) \rho+2 \rho=|j-i| \rho+2 \rho$. Similarly, we can obtain the inequality $\operatorname{dist}\left(x_{i}, x_{j}\right) \leq|j-i| \rho+2 \rho$ for $j+4 \leq i \leq-4$. It follows that $\alpha$ is a quasi-geodesic.

Suppose that there exists $x \in \operatorname{Con}^{\omega}(G, d)$ such that $\operatorname{dist}(x, \operatorname{im}(\alpha)) \geq 2 \rho$. By homogeneity, $\operatorname{Con}^{\omega}(G, d) \backslash B_{\rho}(x)$ has two unbounded components, one of which contains $\operatorname{im}(\alpha)$. As in the proof of Subclaim 3.2, this would imply that $\operatorname{Con}^{\omega}(G, d)$ would have at least three ends.

Thus any asymptotic cone of $\operatorname{Con}^{\omega}(G, d)$ is a line and also an asymptotic cone of $G$, which completes the proof of the proposition.

Lemma 2.17 Suppose that $X$ is an unbounded homogeneous geodesic metric space and $T$ is a vertex homogeneous three valence tree with fixed edge length $\rho$. If $\operatorname{Con}^{\omega}(X, e, d)$ has more than two ends and a global cut-point, then there exists an isometry $f: T \rightarrow \operatorname{Con}^{\omega}(X, e, d)$ such that the components of $T \backslash\{v\}$ map to distinct components of $\operatorname{Con}^{\omega}(X, e, d) \backslash\{f(v)\}$ for every vertex $v$ of $T$.

Proof Fix $\rho>0$. Let $T$ be a vertex homogeneous 3 -valence tree with fixed edge length $\rho$. We will now build an isometry $f: T \rightarrow \operatorname{Con}^{\omega}(X, e, d)$ such that the three components of $T \backslash\{v\}$ map into distinct components of $\operatorname{Con}^{\omega}(X, e, d) \backslash\{f(v)\}$ for every vertex $v$ of $T$. Fix a vertex $v_{0}$ of $T$.

Let $T_{i}$ be a sequence of connected subtrees of $T$ such that $v_{0}=T_{1}, T_{i} \subset T_{i+1}$, $\bigcup_{i} T_{i}=T$ and $T_{i+1}$ has exactly one vertex not contained in $T_{i}$. This implies that $T_{i+1}$ can be obtained from $T_{i}$ be adding exactly one edge and one vertex.

Let $f\left(v_{0}\right)=x_{0}$ for some $x_{0} \in \operatorname{Con}^{\omega}(X, e, d)$. By induction, assume that we have defined $f$ on $T_{i}$ such that $\left.f\right|_{T_{i}}$ is an isometry and $f$ maps the components of $T_{i} \backslash\{v\}$ to distinct components of $\operatorname{Con}^{\omega}(X, e, d) \backslash\{f(v)\}$ for each vertex $v$ of $T_{i}$. Let $e$ be the edge of $T$ that is added to $T_{i}$ to obtain $T_{i+1}$. Then $e$ has exactly one vertex $e^{-}$in $T_{i}$ and one vertex $e^{+}$in $T_{i+1} \backslash T_{i}$. Notice that $T_{i}$ has valence 1 or 2 at $e^{-}$. This implies that $T_{i} \backslash\left\{e^{-}\right\}$and hence $f\left(T_{i} \backslash\left\{e^{-}\right\}\right)$has at most 2 components. Let $C$ be a component of $\operatorname{Con}^{\omega}(X, e, d) \backslash\left\{f\left(e^{-}\right)\right\}$that is disjoint from $f\left(T_{i} \backslash\left\{e^{-}\right\}\right)$. Since all components are unbounded, we may choose a point $x \in C$ such that $\operatorname{dist}\left(x, f\left(e^{-}\right)\right)=\rho$. Let $f\left(e^{+}\right)=x$ and $f(e)$ be a geodesic from $f\left(e^{-}\right)$to $f\left(e^{+}\right)$. It is immediate that the components of $T_{i+1} \backslash\{v\}$ map to distinct components of $\operatorname{Con}^{\omega}(X, e, d) \backslash\{f(v)\}$ for all vertices $v$ in $T_{i+1}$. It only remains to show that $f$ restricted to $T_{i+1}$ is still an 
isometry. This follows trivially from the fact that if $x, y$ are in distinct components of $\operatorname{Con}^{\omega}(X, e, d) \backslash\{z\}$, then $\operatorname{dist}(x, y)=\operatorname{dist}(x, z)+\operatorname{dist}(z, y)$.

This defines a map $f: T \rightarrow \operatorname{Con}^{\omega}(X, e, d)$. Since any two points lie in some $T_{i}$, $f$ is an isometry. We must show that the separation condition is preserved in the limit. Suppose that $v$ is a vertex of $T$ and $T_{i}$ contains the $2 \rho$-neighborhood of $v$. By construction, $f$ takes the components of $T_{i} \backslash\{v\}$ into distinct components of $\operatorname{Con}^{\omega}(X, e, d) \backslash\{f(v)\}$. Notice that each component of $T \backslash\{v\}$ intersects a component of $T_{i} \backslash\{v\}$ nontrivially, which implies that the separation condition still holds.

Corollary 2.18 In addition, $f$ can be chosen such that $f(t)=\left(f_{n}(t)\right)$ for all $t \in T$ where $f_{n}: T \rightarrow X$ takes edges of $T$ to geodesics in $X$.

Proof We will show how to modify the proof of Lemma 2.17. Using the notation from above, we will inductively define $f, f_{n}$ simultaneously. Suppose that $f, f_{n}$ are defined as desired on $T_{i}$. When choosing $x \in C$ we will also fix a representative $\left(x_{n}\right)$ of $x$. Let $f_{n}\left(e^{+}\right)=x_{n}$, which implies that $f\left(e^{+}\right)=\left(f_{n}\left(e^{+}\right)\right)=x$. Let $f_{n}$ map $e$ to any geodesic from $f_{n}\left(e^{-}\right)$to $f_{n}\left(e^{+}\right)$, which implies that $f(e)=\left(f_{n}(e)\right)$ is a geodesic from $f\left(e^{-}\right)$to $f\left(e^{+}\right)$. The rest of the proof remains unchanged.

Proposition 2.19 Suppose that $X$ is an unbounded homogeneous geodesic metric space and $C_{i}$ is a sequence of finite point sets from $\operatorname{Con}^{\omega}(X, e, d)$. Then $\lim _{e}^{\omega} C_{i}$ embeds isometrically into $\operatorname{Con}^{\omega}(X, e, d)$. In addition, if $C_{i}$ is nested, then the canonical copy of $C_{i}$ in $\lim _{e}^{\omega} C_{i}$ is mapped to $C_{i}$.

This proposition was previously shown under the continuum hypothesis by Osin and Sapir and for groups by Sisto [32].

Proof Let $\iota_{i}: C_{i} \rightarrow \operatorname{Con}^{\omega}(X, e, d)$ be the inclusion induced map. Fix a representative for each element of $C=\bigcup_{i} C_{i}$. We can now define a double indexed sequence of maps $\iota_{n}^{i}: C_{i} \rightarrow X$ by letting $i_{n}^{i}(c)$ be the $n^{\text {th }}$ coordinate of our chosen representative for $c \in C$. Thus, if the $C_{i}$ are nested and $c \in C_{i}$, then $\iota_{n}^{j}(c)=\iota_{n}^{i}(c)$ for all $j \geq i$. Hence, $c=\left(l_{n}^{k_{i}}(c)\right)$ for any sequence $k_{i}$. This will imply that the map defined below takes the canonical copy of $C_{i}$ in $\lim ^{\omega} C_{i}$ to $C_{i}$. Let

$$
A_{i}=\left\{n|| \operatorname{dist}\left(c, c^{\prime}\right)-\frac{\operatorname{dist}\left(\iota_{n}^{j}(c), \iota_{n}^{j}\left(c^{\prime}\right)\right)}{d_{n}} \mid \leq \frac{1}{i} \text { for all } c, c^{\prime} \in C_{j} \text { where } j \leq i\right\} .
$$

Since $\left|\bigcup_{j \leq i} C_{j}\right|$ is finite and $\iota_{j}$ is an isometry for every $j, A_{i}$ is $\omega$-large. Let $m_{n}=\max \left\{i \mid n \in A_{i}\right.$ and $\left.i \leq n\right\}$ if this set is non-empty and $m_{n}=1$ otherwise. Suppose that $m_{n}$ was bounded by $L$ on some $\omega$-large set $A$. Then $A_{2 L} \cap A \subset\{1, \ldots, 2 L-1\}$, 
which is a contradiction since $\omega\left(A_{2 L}\right)=\omega(A)=1$ and $\omega(\{1, \ldots, 2 L-1\})=0$. Thus $\lim ^{\omega} m_{n}=\infty$.

Define $\widetilde{\imath}: \lim _{e}^{\omega} C_{i} \rightarrow \operatorname{Con}^{\omega}(X, e, d)$ by $\widetilde{\imath}\left(\left(c_{n}\right)\right)=\left(\iota_{n}^{m_{n}}\left(c_{n}\right)\right)$.

Claim $\tau$ is a well-defined isometric embedding of $\lim _{e}^{\omega} C_{n}$ into $\operatorname{Con}^{\omega}(X, e, d)$.

Fix $c, c^{\prime} \in \lim ^{\omega} C_{n}$. We may choose representatives $c_{n}, c_{n}^{\prime} \in C_{n}$ such that $c=\left(c_{n}\right)$ and $c=\left(c_{n}^{\prime}\right)$. By construction,

$$
\operatorname{dist}\left(c_{n}, c_{n}^{\prime}\right)-\frac{1}{m_{n}} \leq \frac{\operatorname{dist}\left(\iota_{n}^{m_{n}}\left(c_{n}\right), \iota_{n}^{m_{n}}\left(c_{n}^{\prime}\right)\right)}{d_{n}} \leq \operatorname{dist}\left(c_{n}, c_{n}^{\prime}\right)+\frac{1}{m_{n}}
$$

for all $n$ such that $m_{n} \neq 1$. Since $m_{n}$ is $\omega$-divergent, this set is $\omega$-large and

$$
\begin{aligned}
\operatorname{dist}\left(c, c^{\prime}\right) & =\lim ^{\omega}\left[\operatorname{dist}\left(c_{n}, c_{n}^{\prime}\right)-\frac{1}{m_{n}}\right] \leq \lim ^{\omega}\left[\frac{\operatorname{dist}\left(\iota_{n}^{m_{n}}\left(c_{n}\right), \iota_{n}^{m_{n}}\left(c_{n}^{\prime}\right)\right)}{d_{n}}\right] \\
& \leq \lim ^{\omega}\left[\operatorname{dist}\left(c_{n}, c_{n}^{\prime}\right)+\frac{1}{m_{n}}\right]=\operatorname{dist}\left(c, c^{\prime}\right) .
\end{aligned}
$$

Thus $\tau$ is independent of the chosen representative and is an isometry.

We can now use Lemma 2.17 to prove that $\mathbb{R}$-trees can also be transversally embedded into cones with cut-points.

Lemma 2.20 Suppose that $X$ is an unbounded homogeneous geodesic metric space and $T$ is a universal $\mathbb{R}$-tree with continuum branching at every point. If $\operatorname{Con}^{\omega}(X, e, d)$ has more than two ends and a global cut-point, then there exists an isometry $f: T \rightarrow$ $\operatorname{Con}^{\omega}(X, e, d)$ such that the components of $T \backslash\{v\}$ map to distinct components of $\operatorname{Con}^{\omega}(X, e, d) \backslash\{f(v)\}$ for every $v$ in $T$.

Proof Let $T_{i}$ be a three valence tree with edge length $1 / 2^{i}$ such that $T_{i} \subset T_{i+1}$ for all $i \in \mathbb{N}$ and $t_{0}$ a fixed vertex in $T_{1}$. We will assume that $T_{i}$ is endowed with the edge metric. We will use $[v, w]$ to denote the geodesic from $v$ to $w$ in $T_{i}$ and $(v, w)=[v, w] \backslash\{v, w\}$. If $v, w \in T_{i} \cap T_{j}$, then $[v, w]$ is independent of whether the geodesic is taken in $T_{i}$ or in $T_{j}$.

By Lemma 2.17, there exist isometries $f_{i}: T_{i} \rightarrow \operatorname{Con}^{\omega}(X, e, d)$, which satisfy the separation condition of Lemma 2.17. By homogeneity, we may assume $f_{i}\left(t_{0}\right)=f_{j}\left(t_{0}\right)$ for all $i, j$. By Corollary 2.18, there exists a sequence of maps $f_{n}^{i}: T_{i} \rightarrow X$ such that $f_{i}(t)=\left(f_{n}^{i}(t)\right)$ for all $t \in T_{i}$. We will also require that $f_{n}^{i}\left(t_{0}\right)=f_{n}^{j}\left(t_{0}\right)$ for all $i, j$.

Let $V_{i}$ be the vertices of the ball of radius $i$ about $t_{0}$ in $T_{i}$. Then $\left|f_{i}\left(V_{i}\right)\right|$ is a finite set and Proposition 2.19 implies $\lim _{e}^{\omega} f_{i}\left(V_{i}\right)$ embeds isometrically. While $\lim _{e}^{\omega} f_{i}\left(V_{i}\right)$ is 
a universal $\mathbb{R}$-tree, we must still guarantee that the embedding preserves the separation property. To do this we will show how to modify the proof of Proposition 2.19 so as to guarantee that the embedding preserves the desired separation property. Let

$$
A_{i}=\left\{n|| \operatorname{dist}(v, w)-\frac{\operatorname{dist}\left(f_{n}^{j}(v), f_{n}^{j}(w)\right)}{d_{n}} \mid \leq \frac{1}{i} \text { for all } v, w \in V_{j} \text { where } j \leq i\right\} .
$$

For $r=\operatorname{dist}\left(f_{n}^{j}\left(v_{0}\right),\left\{f_{n}^{j}\left(v_{1}\right), f_{n}^{j}\left(v_{2}\right)\right\}\right)$ and $v_{0}, v_{1}, v_{2} \in T_{j}$ such that $v_{0}$ separates $v_{1}$ from $v_{2}$ in $T_{j}$, let

$$
\rho_{n}^{i}\left(j, v_{0}, v_{1}, v_{2}\right)=\operatorname{div}_{1}\left(f_{n}^{j}\left(v_{1}\right), f_{n}^{j}\left(v_{2}\right), f_{n}^{j}\left(v_{0}\right) ; \frac{1}{i r}\right) .
$$

Let

$$
B_{i}=\left\{n \mid \rho_{n}^{i}\left(j, v_{0}, v_{1}, v_{2}\right)>i d_{n} \text { for any } j \leq i, v_{0}, v_{1}, v_{2} \in V_{j} \text { and } v_{0} \in\left(v_{1}, v_{2}\right)\right\} .
$$

As before $A_{i}$ is $\omega$-large for each $i$.

Claim $B_{i}$ is an $\omega$-large set.

For each $j$ and each triple $v_{0}, v_{1}, v_{2} \in V_{j}$ such that $v_{0} \in\left(v_{1}, v_{2}\right)$, we have that

$$
\lim ^{\omega} \frac{\operatorname{div}_{1}\left(f_{n}^{j}\left(v_{1}\right), f_{n}^{j}\left(v_{2}\right), f_{n}^{j}\left(v_{0}\right) ; \frac{1}{2 i r}\right)}{d_{n}}=\infty
$$

by Lemma 2.9 where $r=\operatorname{dist}\left(f_{j}\left(v_{0}\right),\left\{f_{j}\left(v_{1}\right), f_{j}\left(v_{2}\right)\right\}\right)$. Thus

$$
\frac{\operatorname{div}_{1}\left(f_{n}^{j}\left(v_{1}\right), f_{n}^{j}\left(v_{2}\right), f_{n}^{j}\left(v_{0}\right) ; \frac{1}{i r_{n}}\right)}{d_{n}}>i
$$

on an $\omega$-large set where $r_{n}=\operatorname{dist}\left(f_{n}^{j}\left(v_{0}\right),\left\{f_{n}^{j}\left(v_{1}\right), f_{n}^{j}\left(v_{2}\right)\right\}\right)$. Since $V_{j}$ is finite, $B_{i}$ is the finite intersection of $\omega$-large sets, which completes the proof of the claim.

Let $m_{n}=\max \left\{i \mid n \in B_{i} \cap A_{i}\right.$ and $\left.i \leq n\right\}$ if the intersection is non-empty for some $i \leq n$, and $m_{n}=1$ otherwise.

Define $\tilde{t}=\left(t_{0}\right)$ and $\tilde{f}: \lim _{\tilde{t}}^{\omega} T_{i} \rightarrow \operatorname{Con}^{\omega}(X, e, d)$ by $\tilde{f}(t)=\left(f_{n}^{m_{n}}(t)\right)$.

Notice that $\lim _{\tilde{t}}^{\omega} T_{i}=\lim _{\tilde{t}}^{\omega} V_{i}$ and $\tilde{f}\left(\left(t_{o}\right)\right)=f_{i}\left(t_{0}\right)$ for all $i$. As in the proof of Proposition 2.19, $\lim ^{\omega} m_{n}=\infty$ and $\tilde{f}$ is a well-defined isometric embedding of $\lim ^{\omega} T_{i}$ into $\operatorname{Con}^{\omega}(X, e, d)$.

All that remains is to show that $\tilde{f}$ satisfies the desired separation condition. Suppose that $v_{0}, v_{1}, v_{2}$ are points on $\lim _{\tilde{t}}^{\omega} T_{i}$ such that $v_{1}, v_{2}$ are in different components 
of $\lim _{\tilde{t}}^{\omega} T_{i} \backslash\left\{v_{0}\right\}$. Then there exist representatives $\left(v_{n}^{0}\right),\left(v_{n}^{1}\right),\left(v_{n}^{2}\right)$ of $v_{1}, v_{2}, v_{3}$ respectively such that $v_{n}^{1}, v_{n}^{2}$ are in distinct components of $T_{n} \backslash\left\{v_{n}^{0}\right\} \omega$-almost surely. Thus

$$
\frac{\operatorname{div}_{1}\left(f_{n}^{j}\left(v_{n}^{1}\right), f_{n}^{j}\left(v_{n}^{1}\right), f_{n}^{j}\left(v_{n}^{0}\right) ; \frac{1}{m_{n} r_{n}}\right)}{d_{n}}>m_{n}
$$

on an $\omega$-large set where $r_{n}=\operatorname{dist}\left(f_{n}^{j}\left(v_{0}\right),\left\{f_{n}^{j}\left(v_{1}\right), f_{n}^{j}\left(v_{2}\right)\right\}\right)$ and $j \leq m_{n}$. Lemma 2.9 implies that $\tilde{f}\left(v_{1}\right), \tilde{f}\left(v_{2}\right)$ are in distinct components of $\operatorname{Con}^{\omega}(X, e, d) \backslash\left\{\tilde{f}\left(v_{0}\right)\right\}$. This completes the proof.

Proposition 2.21 Let $G$ be a finitely generated group. If $\operatorname{Con}^{\omega}(G, d)$ has a global cut-point, then $\operatorname{Con}^{\omega}(G, d)$ is simply connected or has uncountable fundamental group.

Proof We may assume that $G$ is not virtually cyclic, since the theorem is trivial in that case. Then $G$ has an asymptotic cone $\operatorname{Con}^{\omega}(G, d)$ with a global cut-point and more than two ends. By Lemma 2.20, $\operatorname{Con}^{\omega}(G, d)$ contains an isometrically embedded universal $\mathbb{R}$-tree $T$ such that the components of $T \backslash\{v\}$ map to distinct components of $\operatorname{Con}^{\omega}(G, d) \backslash\{f(v)\}$ where $f$ is the isometric embedding of $T$ into $\operatorname{Con}^{\omega}(G, d)$.

Suppose $\gamma: S^{1} \rightarrow \operatorname{Con}^{\omega}(G, d)$ is an essential loop and fix $x_{0} \in f(T)$, which we may assume is a base point of $\gamma$. Let $\rho=2 \operatorname{diam}(\gamma)$ and $S=\left\{x \in f(T) \mid \operatorname{dist}\left(x, x_{0}\right)=\rho\right\}$. Then $S$ has cardinality continuum and $\operatorname{dist}(x, y)=2 \rho$ for all $x, y \in S$. For $x \in S$, choose $g_{x} \in \prod G$ such that $g_{x} \cdot x_{0}=x$. Let $S_{\gamma}=\left\{g_{x} \cdot \gamma \mid x \in S\right\}$, which is an uncountable set of essential loops in $\operatorname{Con}^{\omega}(G, d)$.

Claim No two loops from $S_{\gamma}$ are homotopic.

Suppose that $g_{x} \cdot \gamma$ is homotopic $g_{y} \cdot \gamma$. Then there exists a continuous map $h: A \rightarrow$ $\operatorname{Con}^{\omega}(G, d)$ of a planar annulus that takes one boundary component to $g_{x} \cdot \gamma$ and the other to $g_{y} \cdot \gamma$. Since $\operatorname{dist}\left(g_{x} \cdot \gamma, x_{0}\right)>0, g_{x} \cdot \gamma$ and $g_{y} \cdot \gamma$ are in distinct components of $\operatorname{Con}^{\omega}(G, d) \backslash\left\{x_{0}\right\}$. Thus $h^{-1}\left(\left\{x_{0}\right\}\right)$ separates the two boundary components of the annulus $A$. Then there exists a single component $C$ of $h^{-1}\left(\left\{x_{0}\right\}\right)$ that separates the boundary components of $A$. This is a consequence of the Phragmén-Brouwer properties (see Hurewicz and Wallman [18]). We can then modify $h$ by mapping the component of the plane bounded by $C$ to $x_{0}$. This is a null homotopy of $g_{x} \cdot \gamma$ that contradicts our choice of $\gamma$ and completes the proof of the claim and theorem.

Corollary 2.22 Let $G$ be a finitely generated group. If $\operatorname{Con}^{\omega}(G, d)$ has a global cut-point, then $\operatorname{Con}^{\omega}(G, d)$ is simply connected or its fundamental group contains an uncountably generated free subgroup. 
Proof Suppose that we have constructed $f: T \rightarrow \operatorname{Con}^{\omega}(G, d), \gamma, S=\{x \in f(T) \mid$ $\left.\operatorname{dist}\left(x, x_{0}\right)=\rho\right\}$ and $S_{\gamma}=\left\{g_{x} \cdot \gamma \mid x \in S\right\}$ as in the proof of Proposition 2.21. Let $p_{x}:[0,1] \rightarrow f(T)$ be the unique geodesic in $f(T)$ from $x_{0}$ to $x \in S$.

Then $S_{\gamma}^{\prime}=\left\{\mathrm{x}=p_{x} * g_{x} \cdot \gamma * \bar{p}_{x} \mid x \in S\right\}$ is a set of loops based at $x_{0}$ (where $\left.\bar{p}_{x}(t)=p_{x}(1-t)\right)$.

Claim $S_{\gamma}^{\prime}$ generates a free product of cyclic groups.

Suppose that $\mathrm{x}_{1}^{n_{1}} * \cdots * \mathrm{x}_{k}^{n_{k}}$ is a null-homotopic loop in $\operatorname{Con}^{\omega}(G, d)$ where $\mathrm{x}_{i} \neq \mathrm{x}_{i+1}$, $\mathrm{x}_{1} \neq \mathrm{x}_{k}$ and $\mathrm{x}_{i}^{n_{i}}$ is an essential loop. Then there exists $h: \mathbb{D} \rightarrow \operatorname{Con}^{\omega}(G, d)$ a map from the unit disc in the plane such that $h(\partial \mathbb{D})$ is a parametrization of the curve $\mathrm{x}_{1}^{n_{1}} *$ $\cdots * \mathrm{x}_{k}^{n_{k}}$. Let $C$ be the closure of the connected component of $h^{-1}\left(\operatorname{Con}^{\omega}(G, d) \backslash\left\{x_{0}\right\}\right)$ containing the subpath $p$ of $\partial \mathbb{D}^{2}$ that maps to $x_{1}^{n_{1}}$. By construction, $\partial \mathbb{D} \cap C=p$ and $h(\partial C \backslash\{p\})=x_{0}$. Define $h^{\prime}: \mathbb{D} \rightarrow \operatorname{Con}^{\omega}(G, d)$ by $h^{\prime}(y)=h(y)$ for $y \in C$ and $h^{\prime}(y)=x_{0}$ for $y \notin C$. Then $h^{\prime}$ is continuous and $\mathrm{x}_{1}^{n_{1}}$ is null-homotopic, which contradicts our choice of $x_{1}^{n_{1}}$. This completes the proof of the claim.

While the subgroup generated by $S_{\gamma}^{\prime}$ may not by a free group ( $\gamma$ might have finite order in the fundamental group), it is the free product of cyclic groups. Thus it is easy to find an uncountably generated free subgroup.

Corollary 2.23 Let $G$ be a finitely generated group. If $G$ is constricted, then every asymptotic cone of $G$ is simply connected or has uncountable fundamental group. If $G$ is not wide, then $G$ has an asymptotic cone that is simply connected or has uncountable fundamental group.

\section{Groups with quasi-isometrically embedded subgroups}

Definition 3.1 A group is a prairie group if all of its asymptotic cones are simply connected.

Lemma 3.2 The following groups are prairie groups:

(1) Nilpotent groups

(2) Hyperbolic groups

(3) Groups with quadratic Dehn functions:

(a) $\mathrm{SL}_{n}(\mathbb{Z})$ for $n \geq 5$

(b) Thompson's group $F$

(c) Mapping class groups

(d) CAT(0) groups

(e) Automatic groups

(f) Baumslag-Solitar groups $\mathrm{BS}_{p p}$, and many, many others 
Proof In [28], Pansu shows that nilpotent groups have a unique asymptotic cone that is homeomorphic to $\mathbb{R}^{n}$ for some $n$. Gromov showed that non-elementary hyperbolic groups have cones that are isometric to a universal $\mathbb{R}$-tree with uncountable branching at every point. Papasoglu in [29] showed that if a group has a quadratic Dehn function then all of its asymptotic cones are simply connected.

Young showed that $\mathrm{SL}_{n}(\mathbb{Z})$ for $n \geq 5$ has a quadratic Dehn function [33]. Guba showed that Thompson's group $F \mathrm{~h}$ as a quadratic Dehn function [17]. Mosher showed that the mapping class groups are automatic [20]. It is shown in [2] that CAT(0) groups have quadratic Dehn functions and in [12] that automatic groups have quadratic Dehn functions.

It is a straightforward exercise using van Kampen diagrams to show that $\mathrm{BS}_{p p}$ has a quadratic Dehn function.

Remark 3.3 In [7], the author and Greg Conner note that such groups are uniformly locally simply connected; specifically, every loop of length $r$ bounds a disc of diameter at most $K r$, where $K$ only depends on the group. However, the discs are not necessarily Lipschitz.

Lemma 3.4 There exists a finitely presented prairie group such that all of its asymptotic cones have uncountable Lipschitz fundamental group.

Proof The discrete Heisenberg group $\langle x, y, z \mid z=[x, y],[x, z]=[y, z]=1\rangle$ is a nilpotent group and hence a prairie group. In fact, every asymptotic cone is homeomorphic to $\mathbb{R}^{3}$. However, it is shown in DeJarnette, Hajlasz, Lukyanenko and Tyson [9, Theorem 4.10] that the Lipschitz fundamental group of the real Heisenberg group is not countable generated.

The key to Proposition 2.21 was that the homotopy between the two loops passed through a cut-point so we could "cut" the homotopy off to build a null homotopy for one of the loops. We will show that the same idea holds if the separating set is a highly connected set instead of a point. To do this we will require the following well-known covering lemma for open sets in the plane. We provide a proof for completeness and to fix notation.

Lemma 3.5 Every bounded open set $U$ of $\mathbb{R}^{2}$ is the union of a null sequence of diadic squares with disjoint interiors. In addition, the squares can be chosen such that if $A_{i}$ is the union of squares with side length at least $1 / 2^{i}$, then $U \backslash A_{i} \subset \mathcal{N}_{\sqrt{2} / 2^{i-1}}(\partial U)$. 
Proof Let $Q_{i}$ be a sequence of partitions of the plane with the Euclidean metric into closed square discs with side length $1 / 2^{i}$ such that $Q_{i}$ refines $Q_{i-1} \cdot Q_{i}$ can be chosen to be the set of squares with vertices

$$
\left\{\left(\frac{j}{2^{i}}, \frac{k}{2^{i}}\right),\left(\frac{j+1}{2^{i}}, \frac{k}{2^{i}}\right),\left(\frac{j+1}{2^{i}}, \frac{k+1}{2^{i}}\right),\left(\frac{j}{2^{i}}, \frac{k+1}{2^{i}}\right) \mid j, k \in \mathbb{Z}\right\} .
$$

Let $D_{0}$ be the maximal subset of $Q_{0}$ such that $A_{0} \subset U$ where $A_{0}=\bigcup_{s \in D_{0}} s$. Then $U \backslash A_{0} \subset \mathcal{N}_{\sqrt{2} / 2^{-1}}(\partial U)$.

We will inductively define $D_{i}$ and $A_{i}$ as follows. Let $D_{i}$ be the maximal subset of $Q_{i}$ such that $\bigcup_{s \in D_{i}} s \subset \overline{U \backslash A_{i-1}}$ where $\overline{U \backslash A_{i-1}}$ is the closure of $U \backslash A_{i-1}$. Let $A_{i}=\left(\bigcup_{s \in D_{i}} s\right) \cup A_{i-1}$. We immediately have $U \backslash A_{i} \subset \mathcal{N} \sqrt{2} / 2^{i-1}(\partial U)$. Then $\bigcup_{i=1}^{\infty} A_{i}=U$.

Definition 3.6 Let $\xi: \mathbb{R}^{+} \rightarrow \mathbb{R}^{+} \cup\{\infty\}$ be a continuous function that vanishes at 0 . Then $\xi$ is a modulus of continuity for $g:(X$, dist $X) \rightarrow(Y$, dist $Y)$ if $\operatorname{dist}_{Y}(g(x), g(y)) \leq$ $\xi\left(\operatorname{dist}_{X}(x, y)\right)$ for all $x, y \in X$.

Let $\left(X\right.$, dist) be a path connected metric space and $\zeta: \mathbb{R}^{+} \rightarrow \mathbb{R}^{+} \cup\{\infty\}$ be an increasing function. We will say that $\zeta$ is a modulus of path-connectivity for ( $X$, dist) if for every pair of points $x, y \in X$ there exists a path $\alpha$ from $x$ to $y$ such that $\operatorname{diam}(\alpha) \leq \zeta(\operatorname{dist}(x, y))$. If $X$ is geodesic than the identity function is a modulus of path-connectivity for $(X$, dist $)$.

Remark 3.7 Let $g:\left(X, \operatorname{dist}_{X}\right) \rightarrow(Y$, dist $Y)$ be a continuous function on a compact metric space $X$. Then $\xi(r)=\sup \left\{\operatorname{dist}_{Y}(g(x), g(y)) \mid \operatorname{dist}_{X}(x, y) \leq r\right\}$ is a modulus of continuity that is finite for every $r$. If $\xi^{\prime}$ is another modulus of continuity for $g$, then $\xi^{\prime}(r) \geq \xi(r)$.

Let $(X$, dist) be a path connected space. Then there exists a modulus of path-connectivity for $X$ that vanishes at 0 if and only if $X$ is uniformly locally path connected.

Lemma 3.8 Suppose that $X$ is a metric space containing a closed, simply connected, uniformly locally path connected and uniformly locally simply connected subset $E$. If $h: A \rightarrow X$ is a continuous map from a planar annulus such that $h^{-1}(E)$ separates the boundary components of $A$, then $h$ takes the boundary components of $A$ to nullhomotopic loops in $X$. 
Proof Let $A=\left\{(x, y) \in \mathbb{R}^{2} \mid \frac{1}{4} \leq x^{2}+y^{2} \leq 1\right\}$ and $\mathbb{D}$ be the unit disc in the plane. It is enough to show that the outer boundary of $A$ maps to a null-homotopic loop. Since $h^{-1}(E)$ separates the boundary components of $A$, a component $C$ of $h^{-1}(E)$ separates the boundaries components of $A$. This follows from the Phragmén-Brouwer properties; see[18]. Let $U$ be the component of $\mathbb{D} \backslash C$ that contains the circle of radius $\frac{1}{2}$. Thus $\partial U \subset A$ and $h(\partial U) \subset E$. Let $\xi$ be a modulus of continuity for $h$.

We can decompose $U$ as a null sequence of diadic squares with disjoint interiors, as in Lemma 3.5. As before, let $A_{i}$ be the union of squares with side length at least $1 / 2^{i}$ that are contained in $U$, and $D_{i}$ the set of squares in $A_{i}$ of side length $1 / 2^{i}$. Then $\bigcup_{i=1}^{\infty} D_{i}$ induces a cellular structure on $U$. We will use $U^{(i)}$ to denote the $i$-skeleton of this cellular structure on $U$. Note this implies that a side of a square in $D_{i}$ is not necessarily an edge but is an edge path.

We will now define a continuous map $g: \mathbb{D} \rightarrow X$ such that $\left.g\right|_{\mathbb{D} \backslash U}=h$. If the boundary of $U$ is a loop, then this is obvious. However, the boundary does not have to be a loop. It can be very complicated (consider the Warsaw circle).

Let $\iota: U \rightarrow \partial U$ be a closest point projection map (which in general will be discontinuous), ie, any map such that $\operatorname{dist}(x, \iota(x)) \leq \operatorname{dist}(x, z)$ for all $z^{\prime} \in \partial U$. For every $x \in U^{(0)}$, let $g(x)=h(\iota(x))$.

Claim If $x \in U^{(0)} \backslash A_{i}$ and $y \in \partial U$, then $\operatorname{dist}(g(x), g(y)) \leq \xi\left(\operatorname{dist}(x, y)+\sqrt{2} / 2^{i-1}\right)$. If $x \in U^{(0)} \backslash A_{i}$ and $y \in \partial U$, then $\operatorname{dist}(x, \iota(x)) \leq \sqrt{2} / 2^{i-1}$. Thus $\operatorname{dist}(\iota(x), y) \leq$ $\operatorname{dist}(x, y)+\sqrt{2} / 2^{i-1}$ and the claim follows.

We now wish to extend $g$ continuously to $\mathbb{D} \backslash U \cup U^{(1)}$. Let $\zeta: \mathbb{R}^{+} \rightarrow R^{+} \cup\{\infty\}$ be a modulus of path-connectivity of $X$ that vanishes at 0 . Then there exists an $\eta>0$ such that $\zeta(t)<\infty$ for all $t<\eta$. Suppose that $e$ is an edge of $U^{(1)}$ with vertices $x, y$ such that $\operatorname{dist}(g(x), g(y))<\eta$. Then there exists a path $\alpha_{x, y}$ in $X$ from $g(x)$ to $g(y)$ such that $\operatorname{diam}\left(\alpha_{x, y}\right) \leq \zeta(\operatorname{dist}(g(x), g(y))$. We may extend $g$ by sending $e$ to $\alpha_{x, y}$. Repeating this for all sufficiently short edges of $U^{(1)}$ and sending the other edges to any path between their end points, we can extend $g$ to $\mathbb{D} \backslash U \cup U^{(1)}$.

Claim $g: \mathbb{D} \backslash U \cup U^{(1)} \rightarrow X$ is continuous.

Suppose that $x_{n}$ is a sequence of points in $U^{(1)}$ such that $x_{n} \rightarrow x_{0}$. If $x_{0} \notin \partial U$, then $x_{n}$ is eventually contained in $A_{i}$ for some $i$ and $g\left(x_{n}\right) \rightarrow g\left(x_{0}\right)$ by the pasting lemma for continuous functions (see Munkres [21]).

If $x_{0} \in \partial U$, then we can choose $x_{n}^{\prime}$ such that $x_{n}, x_{n}^{\prime}$ are contained in a single edge of $D^{(1)}$ and $x_{n}^{\prime} \in U^{(0)}$. Also, we may assume that $x_{n}$ is contained in a sufficiently short 
edge (so as to assume the length condition holds on the edge). Since $x_{n}$ converges to $\partial U$, for every $i$ there exists an $N_{i}$ such that $x_{n} \in U^{(1)} \backslash A_{i}$ for all $n>N_{i}$. Then $\operatorname{dist}\left(x_{n}, x_{n}^{\prime}\right) \leq 1 / 2^{i}$ for all $n>N_{i}$. Thus $\operatorname{dist}\left(g\left(x_{n}\right), g\left(x_{n}^{\prime}\right)\right) \leq \zeta\left(\xi\left(1 / 2^{i}\right)\right)$ for all $n>N_{i}$. Also, the fact that $\operatorname{dist}\left(x_{n}, x_{n}^{\prime}\right) \leq 1 / 2^{i}$ for all $n>N_{i}$ implies that $x_{n}^{\prime}$ converges to $x_{0}$. Then

$$
\begin{aligned}
\operatorname{dist}\left(g\left(x_{0}\right), g\left(x_{n}\right)\right) & \leq \operatorname{dist}\left(g\left(x_{0}\right), g\left(x_{n}^{\prime}\right)\right)+\operatorname{dist}\left(g\left(x_{n}^{\prime}\right), g\left(x_{n}\right)\right) \\
& \leq \xi\left(\operatorname{dist}\left(x_{0}, x_{n}^{\prime}\right)+\sqrt{2} / 2^{i-1}\right)+\zeta\left(\xi\left(1 / 2^{i}\right)\right)
\end{aligned}
$$

for all $n \geq N_{i}$. Thus $\left.g\right|_{\mathbb{D} \backslash U \cup U^{(1)}}$ is continuous, which completes the second claim.

Let $\epsilon_{i}=\max _{s \in D_{i}}\{\operatorname{diam}(g(\partial s))\}$, which is necessarily finite for all $i$. Since $\left.g\right|_{\mathbb{D} \backslash U \cup U^{(1)}}$ is continuous, $\epsilon_{i}$ converges to 0 . Since $E$ is simply connected and uniformly locally simply connected, there exists $\delta_{i}$ such that for every $s \in D_{i}, g(\partial s)$ bounds a disc with diameter at most $\delta_{i}$ where $\delta_{i} \rightarrow 0$ as $i \rightarrow \infty$.

Fix $i>0$ and $s \in D_{i}$. Then we can extend $g$ to all of $s$ by extending $\left.g\right|_{\partial s}$ to a disc with diameter at most $\delta_{i}$.

By doing this process for all $s \in \bigcup_{i \geq 0} D_{i}$, we can extend $g$ to all of $\mathbb{D}$. Repeating the argument from the second claim and using the fact that $\delta_{i} \rightarrow 0$, we can see that this extension is continuous.

An interesting related proposition is the following van Kampen type result for fundamental groups.

Proposition 3.9 Suppose that $X=U \cup V$ is a connected metric space and $U \cap V$ is non-empty, closed, simply connected, uniformly locally path connected and uniformly locally simply connected. Then for $x_{0} \in U \cap V, \pi_{1}\left(V, x_{0}\right) * \pi_{1}\left(U, x_{0}\right)$ canonically embeds into $\pi_{1}\left(X, x_{0}\right)$.

The homomorphism will not necessarily be a surjection. In fact,

$$
\pi_{1}\left(X, x_{0}\right) \backslash\left(\pi_{1}\left(V, x_{0}\right) * \pi_{1}\left(U, x_{0}\right)\right)
$$

will often be uncountable if both $U$ and $V$ are not locally simply connected at $x_{0}$.

The Griffiths space is the wedge of two contractible spaces, which has uncountable fundamental group (Griffiths [15]). The point is that free products only allow for finite products of loops but if the loops are getting small, the fundamental group allows for infinite products. For a rigorous definition of infinite products and further information on this type of phenomenon, see Cannon and Conner [ $4 ; 5]$. 
Proof of Proposition 3.9 Suppose that $f_{i}:(I, 0,1) \rightarrow\left(V, x_{0}, x_{0}\right)$ and $g_{j}:(I, 0,1) \rightarrow$ $\left(U, x_{0}, x_{0}\right)$ are essential loops such that the loop $f_{1} * g_{1} * \cdots * f_{n} * g_{n}$ is null-homotopic in $X$. Let $h: \mathbb{D} \rightarrow X$ be a null homotopy and $C$ a component of $h^{-1}(V)$ containing the portion of $\partial D$ that maps to $f_{1}$. Since $U \cap V$ is path connected and locally path connected, we can define a map $h^{\prime}: C \cup \partial \mathbb{D} \rightarrow V$ such that $\left.h^{\prime}\right|_{C}=h$ and $h^{\prime}(\partial \mathbb{D} \backslash C) \subset U \cap V$. Then, as in Lemma 3.8, $h^{\prime}$ can be extended to a null homotopy of $f_{1}$, which contradicts the assumption that $f_{1}$ was an essential loop.

We will use Ol'shanskii's definitions from [22] for a 0-refinement of a van Kampen diagram, 0-edges and 0-cells, a cancellable pair in a van Kampen diagram, a copy of a cell under 0 -refining, and reduced diagrams. Our definitions of $M$-bands, medians, and boundary paths of $M$-bands will follow those of Ol'shanskii [25].

Definition 3.10 ( $M$-bands) Let $M \subset S \cup\{1\}$ where 1 is the empty word is $S \cup S^{-1}$ and $\Delta$ be a van Kampen diagram over $\langle S \mid R\rangle$. An $M$-edge is an edge in $\Delta$ or $\Gamma(G, S)$ labeled by an element of $M$. An $M$-band $\mathcal{T}$ is a sequence of cells $\pi_{1}, \ldots, \pi_{n}$ in a van Kampen diagram over $\langle S \mid R\rangle$ such that

(i) every two consecutive cells $\pi_{i}$ and $\pi_{i+1}$ in this sequence have a common $M$-edge $e_{i}$ and

(ii) every cell $\pi_{i}, i=1, \ldots, n$ has exactly two $M$-edges, $e_{i-1}$ and $e_{i}$.

Consider lines $l\left(\pi_{i}, e_{i}\right)$ and $l\left(\pi_{i}, e_{i-1}\right)$ connecting a point inside the cell $\pi_{i}$ with midpoints of the $M$-edges of $\pi_{i}$. The broken line formed by the lines $l\left(\pi_{1}, e\right), \ldots$, $l\left(\pi_{i}, e_{i}\right), l\left(\pi_{i}, e_{i-1}\right), \ldots, l\left(\pi_{n}, e_{n}\right)$ is called the median of the band $\mathcal{T}$ and will be denoted by $m(\mathcal{T})$. It connects the midpoints of each $M$-edge and lies inside the union of $\pi_{i}$. We say that an $M$-band is an $M$-annulus if $\pi_{1}$ and $\pi_{n}$ share an $M$-edge. If $\mathcal{T}$ is an $M$-annulus, then the edges $e_{1}$ and $e_{n}$ coincide and $m(\mathcal{T})$ is a simple closed curve. An $M$-band $\mathcal{T}$ will be reduced if no two consecutive cells are inverse images of each other.

Each cell $\pi_{i}$ of an $M$-band $\mathcal{T}$ can be viewed as an oriented 4-gon with edges $e_{i-1}, p_{i}, e_{i}, q_{i}$ where $e_{i-1}, e_{i}$ are $M$-edges of $\pi_{i} ; p_{i}$ begins at the initial vertex of $e_{i-1}$ and ends at the initial vertex of $e_{i}$, and $q_{i}$ begins at the terminal vertex of $e_{i-1}$ and ends at the terminal vertex of $e_{i}$. Then $p_{1} p_{2} \cdots p_{n}$ and $q_{1} q_{2} \cdots q_{n}$ are edge paths in $\Delta$ which we will refer to as the combinatorial boundary paths of $\mathcal{T}$ and denote by $\operatorname{top}_{c}(\mathcal{T})$, $\operatorname{bot}_{c}(\mathcal{T})$ respectively. However, the combinatorial boundary paths can have backtracking in the diagram. The (topological) boundary paths of $\mathcal{T}$ are subpaths of $\operatorname{top}_{c}(\mathcal{T})$ and $\operatorname{bot}_{c}(\mathcal{T})$ obtained by removing all maximal subpaths consisting entirely of backtracking and will be denoted by $\operatorname{top}(\mathcal{T})$ and $\operatorname{bot}(\mathcal{T})$ respectively. While a 
topological boundary path has no backtracking, its label is not necessarily freely reduced. It is also possible that one of $\operatorname{top}(\mathcal{T})$ and $\operatorname{bot}(\mathcal{T})$ is empty.

Let $\mathcal{T}$ be a $M$-annulus in a circular diagram $\Delta . \mathcal{T}$ is a minimal $M$-annulus if there are no $M$-annuli contained in the bounded component of $\mathbb{R}^{2} \backslash m(\mathcal{T})$ where $\Delta$ is considered as a subset of $\mathbb{R}^{2} \cdot \mathcal{T}$ is said to be a maximal $M$-annulus in $\Delta$ if it is not contained in the bounded component of $\mathbb{R}^{2} \backslash m\left(\mathcal{T}^{\prime}\right)$ for any other $M$-annulus $\mathcal{T}^{\prime}$ in $\Delta$. For a more complete description of $M$-bands and their boundaries, see [25].

Definition 3.11 Let $G_{e}$ be an HNN extension of a group $\left\langle A \mid R^{\prime}\right\rangle$ with finitely generated associated subgroups. Then $G_{e}$ has a presentation

$$
\left\langle A, t \mid R^{\prime} \cup \bigcup_{i=1}^{k}\left\{u_{i}^{t}=v_{i}\right\}\right\rangle,
$$

where $\left\{u_{1}, \ldots, u_{k}\right\},\left\{v_{1}, \ldots, v_{k}\right\}$ are generating sets for the associated subgroups $H_{e}=\left\langle u_{i}\right\rangle, K_{e}=\left\langle v_{i}\right\rangle$.

Let $G_{a}$ be an amalgamated product of groups $\left\langle A_{1} \mid R_{1}\right\rangle$ and $\left\langle A_{2} \mid R_{2}\right\rangle$ along $\phi: H_{1} \rightarrow$ $H_{2}$ where $H_{i}$ is a finitely generated subgroup of $\left\langle A_{i} \mid R_{i}\right\rangle$. Then $G_{a}$ has a presentation

$$
\left\langle A_{1}, A_{2} \mid R_{1}, R_{2} \cup \bigcup_{i=1}^{k}\left\{u_{i}=\phi\left(u_{i}\right)\right\}\right\rangle,
$$

where $\left\{u_{1}, \ldots, u_{k}\right\}$ is a generating set for the associated subgroup $H_{1}$.

We will fix the groups $G_{e}$ and $G_{a}$ and their presentations for the remainder of Section 3.

Definition 3.12 Let $H$ be a subgroup of a group $G$ generated by $S$ and $Z, Z^{\prime}$ be subsets of $\Gamma(G, S)$. We will say that $Z, Z^{\prime}$ are $H$-separated if there exists $g \in G$ such that $Z, Z^{\prime}$ are contained in distinct components of $\Gamma(G, S) \backslash g H$ where $g H$ is the set of vertices of $\Gamma(G, S)$ labeled by elements from the coset $g H$.

Lemma 3.13 Let $H$ be a subgroup of a group $G$ generated by $S$. The property of being $H$-separated is invariant under the left action of $G$ on $\Gamma(G, S)$.

Lemma 3.14 Suppose that $H_{e}$ or $K_{e}$ is a proper subgroup of $\left\langle A \mid R^{\prime}\right\rangle$. Let $\gamma$ be a loop in $\Gamma\left(G_{e}, S_{e}\right)$ and $N>\operatorname{diam}(\gamma)$. Then there exist elements $\left\{g_{1}, \ldots, g_{N}\right\}$ in $G_{e}$ such that

(i) $g_{i} \cdot \gamma, g_{j} \cdot \gamma$ are $H$-separated for $H \in\left\{H_{e}, K_{e}\right\}$, and

(ii) $\left|g_{i} g_{j}^{-1}\right| \geq 2 N$ and $\left|g_{i}\right| \leq 4 N$ for all $i \neq j$. 
Proof Without loss of generality, we will assume $K_{e}$ is a proper subgroup. Let $\gamma$ and $N$ be as in the statement of the lemma. Choose $a \in\left\langle A \mid R^{\prime}\right\rangle \backslash K_{e}$ and let $g_{i} \equiv t^{N}(t a)^{i} t^{-N}$. Notice that $g_{i}$ has no pinches for any $i \in \mathbb{Z}$, and $g_{i} g_{j}^{-1}=g_{i-j}$. For $i \neq j,\left|g_{i} g_{j}^{-1}\right|$ is at least $2 N$ since $t^{N}(t a)^{i-j} t^{-N}$ has no pinches. Being $K_{e}-$ separated is invariant under the action of $G_{e}$ on $\Gamma\left(G_{e}, S_{e}\right)$; hence, it is enough to show that $\gamma$ and $g_{i} \cdot \gamma$ are $K_{e}$-separated.

Let $x$ be the vertex of $\Gamma(G, S)$ with label $g_{i}$ and $x_{0}$ the vertex with label 1 .

Since $t^{N}(t a)^{i} t^{-N}$ has no pinches, $g_{i}$ and 1 are contained in distinct components of $\Gamma\left(G_{e}, S_{e}\right) \backslash T^{N+1} K_{e}$ where 1 is the identity element of $G_{e}$. Additionally, $\operatorname{dist}\left(g_{i}\right.$, $\left.T^{N+1} K_{e}\right) \geq N$ and $\operatorname{dist}\left(1, T^{N+1} K_{e}\right) \geq N$. Then $N>\operatorname{diam}(\gamma)$ implies that $\gamma, g_{i} \cdot \gamma$ are in distinct components of $\Gamma\left(G_{e}, S_{e}\right) \backslash T^{N+1} K_{e}$.

An analogous proof gives us the following result for $G_{a}$ where $g_{j}=a_{1}^{N}\left(a_{1} a_{2}\right)^{j} a_{1}^{-N}$ for $a_{i} \in A_{i} \backslash H_{i}$.

Lemma 3.15 Suppose that $H_{i}$ is a proper subgroup of $G_{i}$ for $i=1,2$. Let $\gamma$ be a loop in $\Gamma\left(G_{a}, S_{a}\right)$ and $N>\operatorname{diam}(\gamma)$. Then there exist elements $\left\{g_{1}, \ldots, g_{N}\right\}$ in $G_{a}$ such that

(i) $g_{i} \cdot \gamma, g_{j} \cdot \gamma$ are $H_{1}$-separated and

(ii) $\left|g_{i} g_{j}^{-1}\right| \geq 2 N$ and $\left|g_{i}\right| \leq 4 N$ for all $i \neq j$.

Theorem 3.16 Suppose that $G$ is an HNN extension or amalgamated product where the associated subgroups are proper, quasi-isometrically embedded, prairie groups. Then every asymptotic cone of $G$ is either simply connected or has uncountable fundamental group.

Proof Let $G \in\left\{G_{e}, G_{a}\right\}$ and $S$ be the corresponding generating set for $G$. Suppose that $\operatorname{Con}^{\omega}(G, d)$ is not simply connected. Then there exists $\gamma$ an essential loop in $\operatorname{Con}^{\omega}(G, d)$ and we may choose loops $\gamma_{n}$ in $\Gamma(G, S)$ such that $\left(\gamma_{n}(t)\right)=\gamma(t)$. Let $c_{n}=2 \operatorname{diam}\left(\gamma_{n}\right)$. Let $S_{n}$ be the set of elements of $G$ given by Lemma 3.14 or Lemma 3.15. For every two distinct elements $g_{n}, h_{n}$ of $S_{n}, g_{n} \cdot \gamma_{n}$ and $h_{n} \cdot \gamma_{n}$ are $H$-separated for some quasi-isometrically embedded prairie subgroup $H$ of $G$.

Let $g=\left(g_{n}\right), h=\left(h_{n}\right) \in \prod^{\omega} S_{n}$.

Claim $g \cdot \gamma, h \cdot \gamma$ are well-defined loops in $\operatorname{Con}^{\omega}(G, d)$ and $g \cdot \gamma$ is not homotopic to $h \cdot \gamma$ if $g, h$ are distinct elements of $\prod^{\omega} S_{n}$. 
The first assertion follows from the fact that $g_{n}$ grows big $O$ of the scaling sequence. Suppose that $g \cdot \gamma$ is homotopic to $h \cdot \gamma$ for distinct $h, g$. Then $\omega$-almost surely $g_{n} \neq$ $h_{n}$ and there exists $k_{n}$ such that $g_{n} \cdot \gamma_{n}$ and $h_{n} \cdot \gamma_{n}$ are in distinct components of $\Gamma(G, S) \backslash k_{n} H$.

Thus $g \cdot \gamma, h \cdot \gamma$ are in distinct components of $\operatorname{Con}^{\omega}(G, d) \backslash \lim ^{\omega} k_{n} H$ by Lemma 2.6. Since $H$ is quasi-isometrically embedded, $\lim ^{\omega} k_{n} H$ is bi-Lipschitz to $\operatorname{Con}^{\omega}(H, d)$, which is simply connected, uniformly locally simply connected and geodesic.

Thus $\lim ^{\omega} k_{n} H$ is simply connected, uniformly locally simply connected, and uniformly locally path connected. Hence, Lemma 3.8 implies that $g \cdot \gamma$ and $h \cdot \gamma$ are null-homotopic, which contradicts our choice of $\gamma$.

This completes the proof of the claim. The theorem follows since $\prod^{\omega} S_{n}$ is uncountable.

Corollary 3.17 If $G$ is has more than one end, then every asymptotic cone of $G$ is either simply connected or has uncountable fundamental group.

Proof If $G$ has more than one end, then it has a graph of groups decomposition with finite edge groups and hence is an HNN extension or an amalgamated product with finite associated subgroups, and finite subgroups are always quasi-isometrically embedded prairie groups.

This corollary was also shown in [11] since groups with more than one end are relatively hyperbolic.

The following lemma is due to Burillo.

Lemma 3.18 [3] If $X$ is quasi-isometric to a metric space with a log metric then every asymptotic cone of $X$ is totally disconnected.

Corollary 3.19 Suppose that $G$ is an HNN extension or amalgamated product where the associated subgroups are exponentially distorted. Then every asymptotic cone of $G$ is either simply connected or has uncountable fundamental group.

Proof We will proceed as in the proof of Theorem 3.16. We only need to show how to circumvent the use of Lemma 3.8.

We can construct $S_{n}$ as before and let $g=\left(g_{n}\right), h=\left(h_{n}\right)$ for $g_{n}, h_{n} \in S_{n}$. 
If $g_{n} \neq h_{n} \omega$-almost surely, then there exists $X=\lim ^{\omega} k_{n} H$ such that $g \cdot \gamma, h \cdot \gamma$ are in distinct components of $\operatorname{Con}^{\omega}(G, d) \backslash X$. Since $H$ is exponentially distorted, it is totally disconnected by Lemma 3.18 .

Suppose that $h: A \rightarrow \operatorname{Con}^{\omega}(G, d)$ is a homotopy from $g \cdot \gamma$ to $h \cdot \gamma$. Then there exists a component $C$ of $h^{-1}(X)$ that separates the boundary components of $A$. Since $X$ is totally disconnected, $h(C)$ must be a point. Hence $h$ can be modified to a map on the disc by sending the component of the disc bounded by $C$ to $h(C)$. Thus $g \cdot \gamma$ must be null-homotopic, which contradicts our choice of $\gamma$.

Corollary 3.20 Let $G=\left\langle a, t \mid\left(a^{p}\right)^{t}=a^{q}\right\rangle$ be the Baumslag-Solitar group, where $|p| \neq|q|$. For every $(\omega, d), \operatorname{Con}^{\omega}(G, d)$ has the following properties.

(i) $\operatorname{Con}^{\omega}(G, d)$ is not semilocally simply connected.

(ii) $\pi_{1}\left(\operatorname{Con}^{\omega}(G, d), x_{0}\right)$ is not simple.

(iii) Every decomposition of $\pi_{1}\left(\mathrm{Con}^{\omega}(G, d), x_{0}\right)$ into a free product of subgroups has a factor that is a not free and uncountable.

(iv) $\pi_{1}\left(\operatorname{Con}^{\omega}(G, d), x_{0}\right)$ contains an uncountable free subgroup.

Proof Let $G=\left\langle a, t \mid\left(a^{p}\right)^{t}=a^{q}\right\rangle$ be the Baumslag-Solitar group, where $|p| \neq|q|$. Properties (i)-(iii) are proved in [7, Corollary 3.2]. So we need only prove (iv). The proof is an adaptation of the proof of Corollary 2.22.

Since $\operatorname{Con}^{\omega}(G, d)$ is not semilocally simply connected, it is not simply connected. Thus it contains an essential loop $\gamma$. Theorem 3.16 shows how to find an uncountable set of essential loops, all of which are in distinct components of $\operatorname{Con}^{\omega}(G, d) \backslash \lim _{e}^{\omega} g_{n}\left\langle a^{q}\right\rangle$ for some choice of $g_{n} \in G$.

Using this uncountable set of loops, we can find $S_{\gamma}^{\prime}$ as in Corollary 2.22. We will now use the notation from Corollary 2.22 and show how to modify the proof.

Suppose that $\mathrm{x}_{1}^{n_{1}} * \cdots * \mathrm{x}_{k}^{n_{k}}$ is a null-homotopic loop in $\operatorname{Con}^{\omega}(G, d)$ where $\mathrm{x}_{i} \neq \mathrm{x}_{i+1}$, $\mathrm{x}_{1} \neq \mathrm{x}_{k}$ and $\mathrm{x}_{i}^{n_{i}}$ is an essential loop. Then there exists $h: \mathbb{D} \rightarrow \operatorname{Con}^{\omega}(G, d)$ a map from the unit disc in the plane such that $h(\partial \mathbb{D})$ is a parametrization of the curve $\mathrm{x}_{1}^{n_{1}} * \cdots * \mathrm{x}_{k}^{n_{k}}$. Let $C$ be the closure of the connected component of $\mathbb{D} \backslash h^{-1}\left\{\lim _{e}^{\omega} g_{n}\left\langle a^{q}\right\rangle\right\}$ containing the subpath $p$ of $\partial \mathbb{D}$ that maps to $\mathrm{x}_{1}^{n_{1}}$.

Recall that $\left\langle a^{q}\right\rangle$ is exponential distorted in $G$. Thus $\lim _{e}^{\omega} g_{n}\left\langle a^{q}\right\rangle$ is totally disconnected by Lemma 3.18.

Since $C$ is the closure of a component of $\mathbb{D} \backslash h^{-1}\left(\left\{\lim _{e}^{\omega} g_{n}\left\langle a^{q}\right\rangle\right\}\right), \partial C \backslash\{p\}$ is connected and maps into $\lim _{e}^{\omega} g_{n}\left\langle a^{q}\right\rangle$. Hence $h(\partial C \backslash\{p\})$ is a point $b$. 
Define $h^{\prime}: \mathbb{D} \rightarrow \operatorname{Con}^{\omega}(G, d)$ by $h^{\prime}(y)=h(y)$ for $y \in C$ and $h^{\prime}(y)=b$ for $y \notin C$. Then $h^{\prime}$ is continuous and $\mathrm{x}_{1}^{n_{1}}$ is null-homotopic, which contradicts our choice of $x_{1}^{n_{1}}$. Again, the subgroup generated by $S_{\gamma}^{\prime}$ may not by a free group but it is the free product of cyclic groups. Thus it is easy to find an uncountably generated free subgroup. This completes the proof of the corollary.

\subsection{Partitions of van Kampen diagrams}

The following definitions of partitions are due to Papasoglu in [29].

Partitions of the unit disc in the plane Let $D$ be the unit disk in $\mathbb{R}^{2}$ or the planar annulus $\left\{(x, y) \mid x^{2}+y^{2} \in\left[\frac{1}{4}, 1\right]\right\}$. A partition $P$ of $D$ is a finite collection of closed discs $D_{1}, \ldots, D_{k}$ in the plane with pairwise disjoint interiors such that $D=\bigcup_{i} D_{i}$, $\partial D=\partial\left(D_{1} \cup \cdots \cup D_{k}\right)$ and $D_{i} \cap D_{j}=\partial D_{i} \cap \partial D_{j}$ when $i \neq j$. A point $p$ on $\partial D_{1} \cup \cdots \cup \partial D_{k}$ is called a vertex of the partition if for every open set $U$ containing $p$, $U \cap\left(\partial D_{1} \cup \cdots \cup \partial D_{k}\right)$ is not homeomorphic to an interval. An edge of a partition is a pair of adjacent vertices of a disc in the partition. A piece of a partition is the set of the vertices of a disc in the partition. A partition is then a cellular decomposition of the underline space of $P$ where each vertex has degree at least 3, so we will use the standard notation, $P^{(i)}$, to denote the $i^{\text {th }}$ skeleton of a partition.

Geodesic $n$-gons in a metric space $X$ An $n$-gon in $X$ is a map from the set of vertices of the standard regular $n$-gon in the plane into $X$, ie, an ordered set of $n$ points in $X$. If $X$ is a geodesic metric space, we can extend the $n$-gon to edges by mapping the edge between adjacent vertices of the standard regular $n$-gon in the plane to a geodesics segment joining the corresponding vertices of the $n$-gon in $X$. We will say that such an extension is a geodesic $n-$ gon in $X$.

Partitions of loops in a geodesic metric space $X$ Let $\mathbb{D}$ be the unit disc in the plane and $\gamma: \partial \mathbb{D} \rightarrow X$ be a continuous map. A partition of $\gamma$ is a map $\Pi$ from the set of vertices of a partition $P$ of $\mathbb{D}$ to $X$ such that

$$
\left.\Pi\right|_{\partial P \cap P^{(0)}}=\left.\gamma\right|_{\partial P \cap P^{(0)}} .
$$

The vertices, edges, and pieces of $\Pi$ are the images of vertices, edges, and pieces of $P$, respectively. We will write $\Pi\left(\partial D_{i}\right)$ for the pieces of $\Pi$, where the $D_{i}$ are the 2-cells of the partition $P$.

Remark 3.21 Suppose that $\Pi: P^{(0)} \rightarrow X$ is a partition of a loop $\gamma$ in a geodesic metric space. We can extend $\Pi$ to $P^{(1)}$ by mapping every edge contained in $\partial P^{(2)}$ to the corresponding subpath of $\gamma$ and every edge not contained in $\partial P^{(2)}$ to a geodesic 
segment joining its endpoints. Then the length of a piece is the arc length of the loop $\Pi\left(\partial D_{i}\right)$. We will write $\left|\Pi\left(\partial D_{i}\right)\right|$ for the length of the piece $\Pi\left(\partial D_{i}\right)$. We define the mesh of $\Pi$ by

$$
\operatorname{mesh}(\Pi)=\max _{1 \leq i \leq k}\left\{\left|\Pi\left(\partial D_{i}\right)\right|\right\}
$$

At times it will be convenient to ignore some pieces of a partition. If $Z$ is a subset of the pieces of $P$, then the relative mesh of $\Pi$ is

$$
\operatorname{rmesh}_{Z}(\Pi)=\max _{D_{i} \in Z}\left\{\left|\Pi\left(\partial D_{i}\right)\right|\right\}
$$

When $X$ is a Cayley graph of a group, we will also assume that the partition takes vertices of $P$ to vertices in the Cayley graph. A partition $\Pi$ is called a $\delta$-partition if mesh $\Pi<\delta$. A loop of length $k$ in a geodesic metric space is partitionable if it has a $\frac{k}{2}$-partition.

Let $P(\gamma, \delta)$ be the minimal number of pieces in a $\delta$-partition of $\gamma$ if a $\delta$-partition exist and infinity otherwise.

If $P$ is a partition of the unit disc in the plane, then $P^{(1)}$ can be considered as a planar graph where every vertex has degree at least 3 . Then one can use the fact that the Euler characteristic of a planar graph is 1 to obtain the following.

Lemma 3.22 Let $\Pi: P^{(1)} \rightarrow \Gamma(G, S)$ be a partition of a loop in the Cayley graph of $G$. If $\Pi$ has $F$ pieces, then $\Pi$ has at most $3 F$ edges and at most $2 F$ vertices.

A straightforward inductive argument gives us the following lemma.

Lemma 3.23 Suppose that $T$ is a finite simplicial tree with at most $j$ vertices of degree 1 . Then $T$ has at most $j-1$ vertices with degree greater than 2 .

Our goal for the remainder of Section 3.1 and Section 4 is to define partitions of van Kampen diagrams and show how to use the standard techniques for reducing van Kampen diagrams to build nice partitions of loops in the Cayley graph.

Definition 3.24 Suppose that $\langle A, t \mid R\rangle$ is an HNN extension with stable letter $t$. Let $w$ be a word in the alphabet $S \cup S^{-1}$. We will use $|w|_{F}$ to denote the freely reduced word length of $w,|w|_{G}$ to denote the minimal word length of $w$ in $\langle A, t \mid R\rangle$ and $|w|_{t}$ to denote the number of $t$-letters in $w$.

A word $w$ is a $t$-shortest word if $|w|_{t} \leq\left|w^{\prime}\right|_{t}$ for all $w^{\prime}={ }_{G} w$ and

$$
|w|_{G}=|w|_{t}+\sum\left|v_{i}\right|_{G}
$$


where $v_{i}$ ranges over maximal $a$-subwords of $w$. To avoid trivialities, we will also require that every $a$-subword of a $t$-shortest word be freely reduced.

We will say that $w$ is an almost $t$-shortest word if $|w|_{t} \leq\left|w^{\prime}\right|_{t}$ for all $w^{\prime}={ }_{G} w$.

A path $\gamma$ in the Cayley graph of $G$ is a $t$-shortest path (or an almost $t$-shortest path) if $\mathbf{L a b}(\gamma)$ is a $t$-shortest word (or an almost $t$-shortest word).

The equality in (2) implies that if we replace each maximal $a$-subword of a $t$-shortest path with a geodesic, then the whole path is geodesic. This gives us the following result.

Lemma 3.25 Every edge in $\Gamma(G, S)$ labeled by a $t$-letter on a $t$-shortest path from $g$ to $h$ is also an edge of a geodesic from $g$ to $h$.

Definition 3.26 Let $P$ be a partition of the unit disc $\mathbb{D}^{2}$ or the unit annulus in the plane and $\Delta$ a van Kampen diagram over $\langle S \mid R\rangle$. A continuous map $\Psi: P^{(2)} \rightarrow \Delta$ is a partition of $\Delta$ if it satisfies the following conditions.

(i) $\Psi\left(P^{(0)}\right) \subset \Delta^{(0)}$.

(ii) $\Psi$ takes edges of $P$ to edge paths in $\Delta^{(1)}$.

(iii) For each closed 2-cell $D$ of $P, \Psi(D)$ is a reduced subdiagram of $\Delta$.

If we consider $\Delta$ as a metric space with the edge metric, then $\left.\Psi\right|_{P^{(0)}}$ is a partition of the loop $\partial \Delta$ under our previous definition.

As before, the edges, vertices and pieces of $\Psi$ are the images under $\Psi$ of edges, vertices and pieces of $P$ in $\Delta$, respectively.

Define the mesh of $\Psi$ by $\operatorname{mesh}(\Psi)=\operatorname{mesh}(\theta \circ \Psi)$ where $\theta$ is the canonical map into the Cayley complex.

$\Psi$ is an $h$-partition of $\Delta$ if $\Psi$ is partition of $\Delta$ and a homeomorphism. If $\Psi$ is a $h$-partition of $\Delta$ and $\theta \circ \Psi$ takes edges of $P$ to geodesic paths ( $t$-shortest paths), then we will say $\Psi$ is a geodesic partition ( $t$-shortest partition) of $\Delta$.

This gives the underling space of $\Delta 2$-cell structures, the cell structure inherited as a van Kampen diagram and the cell structure inherited from the partition. When there is a chance of confusion, we will specify if we are considering a vertex/edge in the underlying space as a $\Psi$-vertex $/ \Psi$-edge or a $\Delta$-vertex/ $\Delta$-edge.

The following lemma follows trivially by considering each of the three types of 0 refinements.

Lemma 3.27 Suppose that $\Psi: P^{(2)} \rightarrow \Delta$ is a partition (or a geodesic partition) and $\Delta^{\prime}$ is a 0 -refinement of $\Delta$. Then there exists a partition (or geodesic partition) $\Psi: P^{(2)} \rightarrow \Delta^{\prime}$, which preserves the number of pieces, edges and vertices, the mesh of the partition, and the labels of edges (after removing any possible 1 's). 


\section{HNN extensions with free associated subgroups}

Let $G$ be a multiple HNN extension of a free group $F$ with free associated subgroups. Then $G$ has a presentation

$$
\left.\left\langle A \cup\left\{t_{i}\right\}\right|\left\{u_{i, s}^{t_{i}}=v_{i, s}\right\}, \quad \text { for } i=1, \ldots, k \text { and } s=1, \ldots, j_{i}\right\rangle,
$$

where $U_{i}=\left\langle u_{i, 1}, \ldots, u_{i, j_{i}}\right\rangle, V_{i}=\left\langle v_{i, 1}, \ldots, v_{i, j_{i}}\right\rangle$ are free subgroups with free generating sets $\left\{u_{i, j}\right\},\left\{v_{i, j}\right\}$ respectively and $t_{i}$ are stable letters. We will use $\langle S \mid R\rangle$ to denote this presentation for $G$ which we will fix throughout Section 4. Let

$$
K=\max \left\{\left|u_{i, 1}\right|_{F}, \ldots,\left|u_{i, j_{i}}\right|_{F},\left|v_{i, 1}\right|_{F}, \ldots,\left|v_{i, j_{i}}\right|_{F}\right\} .
$$

We will also fix the constant $K$ throughout this section. To simplify notation, we will frequently refer to $t_{i}$-bands in diagrams over $\langle S \mid R\rangle$ as just $t$-bands when the specific $i$ is inconsequential.

Lemma 4.1 Let $\mathcal{T}$ be a $t$-band in a van Kampen diagram $\Delta$. Then $\Delta$ can be modified while preserving the numbers of cells and the boundary label of $\Delta$ such that the labels of $\operatorname{top}(\mathcal{T})$ and $\operatorname{bot}(\mathcal{T})$ are freely reduced words.

Proof If $\operatorname{Lab}(\operatorname{bot}(\mathcal{T}))=w_{1} u u^{-1} w_{2}$, then we may cut $\Delta$ along the subpath of $\operatorname{bot}(\mathcal{T})$ labeled by $u u^{-1}$ and re-identify them as in Figure 1 . This is the so called diamond move (see Collins and Huebschmann [6]). A similar process can be performed for $\operatorname{top}(\mathcal{T})$.
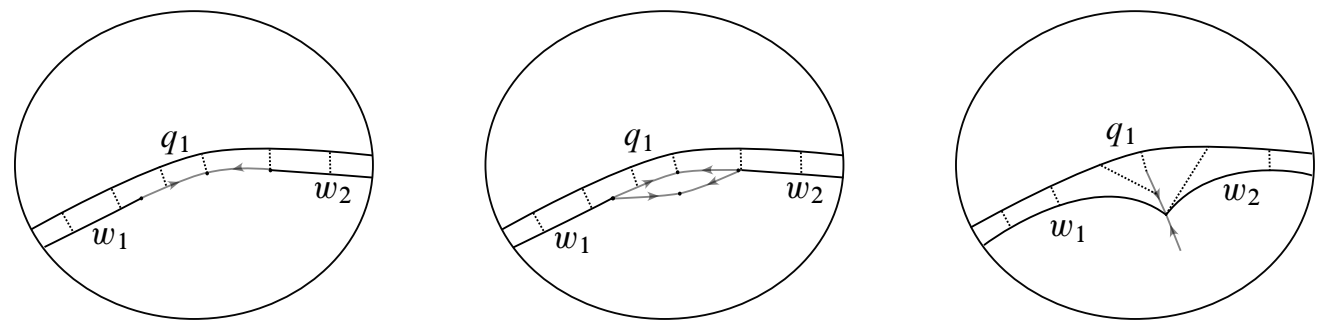

Figure 1: Modifying $\Delta$ to insure that the label of the boundary of a $t$-band is freely reduced. Edges in gray are labeled by $u$ or $u^{-1}$ respectively.

Lemma 4.2 Suppose that $\mathcal{T}$ is a reduced $t$-band in a van Kampen diagram $\Delta$ over $\langle S \mid R\rangle$ endowed with the edge metric. Then there exists an $L$ such that $\operatorname{top}(\mathcal{T})$ is in the $L$-neighborhood of $\operatorname{bot}(\mathcal{T})$ where $L$ is a constant depending only on the associated subgroups. 
Proof The lemma is trivial if you are considering $\operatorname{top}_{c}(\mathcal{T})$ and $\operatorname{bot}_{c}(\mathcal{T})$ in place of $\operatorname{top}(\mathcal{T})$ and $\operatorname{bot}(\mathcal{T})$. So we will prove the lemma by finding a bound on the diameter of the backtracking that was removed to obtain $\operatorname{top}(\mathcal{T})$.

Recall that $\left\{u_{i, 1}, \ldots, u_{i, j_{i}}\right\},\left\{v_{i, 1}, \ldots, v_{i, j_{i}}\right\}$ are free generating sets for the associated subgroups where $u_{i, k}, v_{i, k}$ are words in the alphabet $A$. For the purposes of this lemma, let $U$ be the disjoint union of $\left\langle u_{i, 1}, \ldots, u_{i, j_{i}}\right\rangle$ and $\left\langle v_{i, 1}, \ldots, v_{i, j_{i}}\right\rangle$ and if $g \in U$, let $|g|_{s}$ denote the length in the associated subgroup. Let

$$
L^{\prime}=\max \left\{\left.|g|_{s}|g \in U,| g\right|_{G} \leq 2 K\right\} .
$$

Fix $\mathcal{T}$ a reduced $t$-band in $\Delta$ and $v$ a vertex on $\operatorname{top}(\mathcal{T})$. Then there exists a vertex $v^{\prime}$ on $\operatorname{bot}_{c}(\mathcal{T})$ such that $\operatorname{dist}\left(v, v^{\prime}\right) \leq K+1$. Suppose that $p$ is a maximal subpath of $\operatorname{bot}_{c}(\mathcal{T})$ that contains the vertex $v^{\prime}$ and has freely trivial label in $F(A)$. We will assume (without loss of generality) that $\operatorname{bot}_{c}(\mathcal{T})$ is labeled by words from $\left\{u_{i, 1}, \ldots, u_{i, j_{i}}\right\}$. Then for some $j$,

$$
\mathbf{L a b}(p)=w_{1} u_{j, s_{1}}^{\epsilon_{1}} \cdots u_{j, s_{r}}^{\epsilon_{r}} w_{2}
$$

where $w_{1}$ is a terminal segment of $u_{j, s_{0}}^{\epsilon_{0}}, w_{2}$ is an initial segment of $u_{j, s_{r+1}}^{\epsilon_{r+1}}$, and $\epsilon_{i}= \pm 1$. Let $g=u_{j, s_{0}}^{\epsilon_{0}} \cdots u_{j, s_{r+1}}^{\epsilon_{r+1}}$. By construction $|g|_{G}$ is at most $2 K$ and in $U$. Thus $|g|_{s} \leq L^{\prime}$. This implies that $v^{\prime}$ is at most $L^{\prime} K$ from a vertex $\operatorname{bot} \operatorname{bot}(\mathcal{T})$.

Thus $v$ is at most $L=L^{\prime} K+K+1$ from a vertex of $\operatorname{bot}(\mathcal{T})$, which completes the lemma.

The following lemma is a correction of a lemma by Ol'shanskii and Sapir in [24].

Lemma 4.3 There exists a constant $L$ such that every diagram over $\langle S \mid R\rangle$ that has no $t$-annuli and where all $t$-bands are reduced has diameter no greater than $3 L|\partial \Delta| / 2$.

Proof Let $L$ be the constant from Lemma 4.2.

Let $s$ be the number of $t$-bands in $\Delta$ and $n=|\partial \Delta|$. Then $s \leq n / 2$. There exists a $t$-band $\mathcal{T}$ such that (without loss of generality) $\operatorname{top}_{c}(\mathcal{T}$ ) is contained in $\partial \Delta$ (see [24, Lemma 2.1]). Then $\Delta$ is obtained by gluing $\mathcal{T}$ and a diagram $\Delta_{1}$ with $s-1 t$-bands that satisfy the same hypothesis. Every vertex on a $\operatorname{bot}(\mathcal{T})$ can be connected to the boundary of $\Delta$ by a path of length at most $L$. By induction on $s$, we can deduce that every vertex inside $\Delta$ can be connected to the boundary of $\Delta$ by a path of length at most $L s \leq L n / 2$. Hence the diameter of $\Delta$ is at most $3 L n / 2$. 
Lemma 4.4 Let $\Delta$ be a van Kampen diagram with no $t$-annuli, every $t$-band reduced, and $\gamma:[0,1] \rightarrow \Delta$ be a parametrization of $\partial \Delta$. Suppose that $0=t_{0}<t_{1}<\cdots<t_{k}<$ $t_{k+1}=1$ is a partition of the unit interval and $I$ a subset of $\{0, \ldots, k\}$ such that $\gamma$ restricted to $\left[t_{i}, t_{i+1}\right]$ is a $t$-shortest path for $i \in I$. Then $\theta(\Delta)$ has diameter no greater than

$$
\frac{5 L}{2}\left(\sum_{i \notin I}|\gamma|_{\left[t_{i}, t_{i+1}\right]} \mid+\sum_{i \in I} \operatorname{dist}\left(\theta \circ \gamma\left(t_{i}\right), \theta \circ \gamma\left(t_{i+1}\right)\right)\right),
$$

where $L$ is the constant for Lemma 4.2 and $\theta$ is the canonical map into the Cayley graph.

Proof Let $C=\sum_{i \notin I}|\gamma|_{\left[t_{i}, t_{i+1}\right]} \mid+\sum_{i \in I} \operatorname{dist}\left(\theta \circ \gamma\left(t_{i}\right), \theta \circ \gamma\left(t_{i+1}\right)\right)$. By the same argument as in Lemma 4.3, every vertex of $\Delta$ can be connected to a vertex on $\partial \Delta$ by a path of length at most $L s$ where $s$ is the number of $t$-bands in $\Delta$.

For $i \in I$, let $w_{i}=\mathbf{L a b}\left(\left.\gamma\right|_{\left[t_{i}, t_{i+1}\right]}\right)$ and $\tilde{w}_{i}$ be a geodesic word obtained by replacing each maximal $a$-subpath of $w_{i}$ by a geodesic word. For $i \notin I$, let $w_{i}=$ $\operatorname{Lab}\left(\left.\gamma\right|_{\left[t_{i}, t_{i+1}\right]}\right)=\tilde{w}_{i}$. Then $C=\left|\tilde{w}_{0} \tilde{w}_{1} \cdots \tilde{w}_{k}\right|$. Fix $\Delta_{i}$ a reduced van Kampen diagram with $\partial \Delta_{i}=p_{i} \tilde{p}_{i}$ where $\operatorname{Lab}\left(p_{i}\right)=w_{i}$ and $\mathbf{L a b}\left(\tilde{p}_{i}\right)=\tilde{w}_{i}^{-1}$. Let $s_{i}$ be the number of $t$-bands in $\Delta_{i}$. Since no $t$-band of $\Delta_{i}$ can start and stop on $p_{i}, s, s_{i} \leq C / 2$. By repeating the arguments from Lemma 4.3, we can see that any point in $\Delta_{i}$ is at most $L s_{i}$ from a point on $\tilde{p}_{i}$. Hence, if $x, y$ are two points on $\partial \Delta$, then

$$
\operatorname{dist}(\theta(x), \theta(y)) \leq L s_{i}+L s_{j}+\frac{C}{2} \leq \frac{L C}{2}+\frac{L C}{2}+\frac{C}{2} .
$$

If $x, y$ are two points in $\Delta$,

$$
\operatorname{dist}(\theta(x), \theta(y)) \leq 2(L s)+\left(L s_{i}+L s_{j}+\frac{C}{2}\right) .
$$

Therefore

$$
\theta(\Delta) \leq L C+\left(L C+\frac{C}{2}\right) \leq \frac{5 L C}{2} .
$$

Remark 4.5 Let $\Pi: P^{(0)} \rightarrow \Gamma(G, S)$ be a partition of a loop $\gamma$ in $\Gamma(G, S)$. We can extend $\Pi$ to $P^{(1)}$ as in Remark 3.21, but instead of mapping the interior edges of $P$ to geodesics, we will map the interior edges to $t$-shortest paths in $\Gamma(G, S)$. We can label the edges of $P^{(1)}$ with the labels of their images. Then we can fill each piece with a reduced circular van Kampen diagram. This produces a van Kampen diagram with boundary label equal to the Lab $(\gamma)$ and $\Pi$ induces a canonical homeomorphism from $P^{(2)}$ onto this van Kampen diagram. Thus every partition $\Pi$ of $\gamma$ induces a $t$-shortest partition $\Psi$ of a diagram such that $\Pi=\theta \circ \Psi$. Then by Lemma 4.4, each subdiagram corresponding to a piece has diameter at most $5 \mathrm{Ln} / 2$. 


\subsection{Removing $t$-bands from partitions}

Definition 4.6 Suppose $\Psi: P^{(2)} \rightarrow \Delta$ is a $t$-shortest partition of a van Kampen diagram $\Delta$. A $t$-band $\mathcal{T}$ crosses a $\Psi$-edge $e$ if $e$ contains a $t$-edge from $\mathcal{T}$. If $\mathcal{T}$ is a $t$-annulus which crosses a $\Psi$-edge $e$, we will call the endpoints of the corresponding $t$-edge the crossing vertices of $\mathcal{T}$.

Lemma 4.7 If $\Psi$ is a $t$-shortest partition of $\Delta$ and $\mathcal{T}$ is a $t$-band in $\Delta$, then $\mathcal{T}$ crosses each $\Psi$-edge at most once.

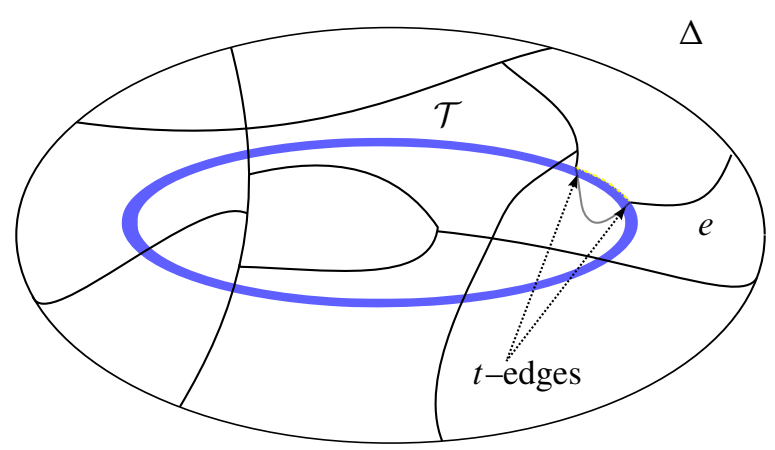

Figure 2: A $\Psi$-edge that crosses $\mathcal{T}$ twice cannot be $t$-shortest.

Proof If $\mathcal{T}$ crossed a $\Psi$-edge $e$ twice, then $e$ would contain two $t$-edges and the subword of $e$ beginning and ending with these $t$-edges would be equal to a subword of $\operatorname{top}_{c}(\mathcal{T})$ or $\operatorname{bot}_{c}(\mathcal{T})$ (see Figure 2). Thus $e$ was not $t$-shortest. (Note we are using the fact the diagrams are planar.)

Corollary 4.8 Let $\Psi: P^{(2)} \rightarrow \Delta$ be a $t$-shortest partition of $\Delta$ and $\mathcal{T}$ be a $t$-annulus in $\Delta$. Then the bounded component of $P^{(2)} \backslash \Psi^{-1}(m(\mathcal{T}))$ contains a vertex of $P$.

Corollary 4.9 Let $\Psi: P^{(2)} \rightarrow \Delta$ be a $t$-shortest partition of $\Delta$. Then $\Delta$ can have at most $V$ maximal $\mathcal{T}$-annuli, where $V$ is the number of vertices of the partition $\Psi$.

Lemma 4.10 Let $\Psi: P^{(2)} \rightarrow \Delta$ be an $h$-partition of $\Delta$ with $F$ pieces, where $\Delta$ is an annular diagram where the boundary components have labels that are trivial in $G$. Suppose that $\mathcal{T}$ is a $t$-annulus in $\Delta$ such that $\mathcal{T}$ crosses each edge at most once and if $v$ is a crossing vertex of a $\Psi-$ edge with vertices $e_{-}, e_{+}$, then $\operatorname{dist}\left(e_{-}, e_{+}\right) \leq$ $\operatorname{dist}\left(e_{-}, v\right)+\operatorname{dist}\left(v, e_{+}\right)$. Let $B=\max _{D \in P}\{\operatorname{diam}(\theta \circ \Psi(D))\}$. 
Then there exists a partition $\widetilde{\Psi}: \widetilde{P}^{(2)} \rightarrow \Delta^{\prime}$, where $\Delta^{\prime}$ is obtained by removing $\mathcal{T}$ such that:

(i) $\widetilde{\Psi}$ has no more than $9 F^{2}+4 F$ pieces, and

(ii) $\operatorname{mesh}(\widetilde{\Psi}) \leq \max \{3(B+2 K)$, $\operatorname{mesh}(\Psi)\}$,

where $K$ is the max of the word length of the generators of the associated subgroups.

Proof Let $\Psi: P^{(2)} \rightarrow \Delta$ be a partition of $\Delta$ as in the statement of the lemma and let $A$ be the underline space of $P$. Let $\Delta_{A}$ be the subdiagram of $\Delta$ obtained by removing all cells interior to $\operatorname{top}_{c}(\mathcal{T})$.

Let $V=\left\{v_{1}, v_{2}, \ldots, v_{k}\right\}$ be the set of crossing vertices of $\mathcal{T}$ that are contained in $\operatorname{top}_{c}(\mathcal{T})$ where the ordering is obtained by traversing $\operatorname{top}_{c}(\mathcal{T})$ in the clockwise direction. Let $q_{i}$ be a subpath of $\operatorname{top}_{c}(\mathcal{T})$ between $v_{i}$ and $v_{i+1}$ without backtracking (where the indices are taken modulo $k$ ) that intersects $V$ only at $v_{i}, v_{i+1}$ and $m\left(q_{i}\right)$ the corresponding subpath of $m(\mathcal{T})$. Since $\mathcal{T}$ crosses each vertex at most once, $k \leq 3 F$.

By construction, $m\left(q_{i}\right)$ is contained inside of $\Psi(D)$ for some piece $D$ of $P$. Thus $q_{i}$ is in the $K$-neighborhood of $\Psi(D)$ and $\operatorname{diam}\left(\theta \circ \Psi\left(q_{i}\right)\right) \leq B+2 K$.

Claim 4 There exists a refinement $P^{\prime}$ of $P$ and a partition $\Psi^{\prime}: P^{\prime(2)} \rightarrow \Delta$ with $\Psi^{\prime}(x)=\Psi(x)$ for all $x \in P^{(1)}$ such that:

(i) The number of pieces of $P^{\prime}$ is less than $4 F$.

(ii) $\operatorname{mesh}\left(\Psi^{\prime}\right) \leq \operatorname{mesh}(\Psi)$.

(iii) There is a simple closed curve $\beta_{\mathcal{T}}$ in $P^{\prime(1)}$ such that

(a) $\Psi\left(\beta_{\mathcal{T}}\right) \subset \operatorname{top}_{c}(\mathcal{T})$.

(b) $\beta_{\mathcal{T}}$ has at most $3 F$ edges.

(c) If $\Psi^{\prime}(x)$ is interior to $m(\mathcal{T})$, then $x$ is interior to $\beta_{\mathcal{T}}$.

Proof of Claim 4 Let $w_{i}=\Psi^{-1}\left(v_{i}\right)$ and $W=\left\{w_{i}\right\}$. For each pair $i$, there exists a unique cell $D_{i}$ of $P$ such that $\Psi^{-1}\left(m\left(q_{i}\right)\right) \subset D_{i}$. Let $e_{i}$ be an arc in $D_{i}$ from $w_{i}$ to $w_{i+1}$ such that $e_{i} \cap P^{(1)}=\left\{w_{i}, w_{i+1}\right\}$. In addition, we may assume that the arcs $e_{i}$ have disjoint interiors. Then $\beta_{\mathcal{T}}=e_{1} * e_{2} * \cdots * e_{k}$ is a simple closed curve.

Let $P^{\prime(0)}=P^{(0)} \cup W$. The edges of $P^{\prime}$ are the closure of the connected subsets of $P^{(1)} \cup \beta_{\mathcal{T}} \backslash P^{\prime(0)}$. This gives $\beta_{\mathcal{T}}$ a cellular structure. Each vertex of $\beta_{\mathcal{T}}$ corresponds to a crossing vertex of $\partial_{o} \mathcal{T}$. Since $\beta_{\mathcal{T}}$ has at most $3 F$ vertices and each edge cuts a piece of $P$ into two pieces, $P^{\prime}$ has at most $4 F$ pieces. 


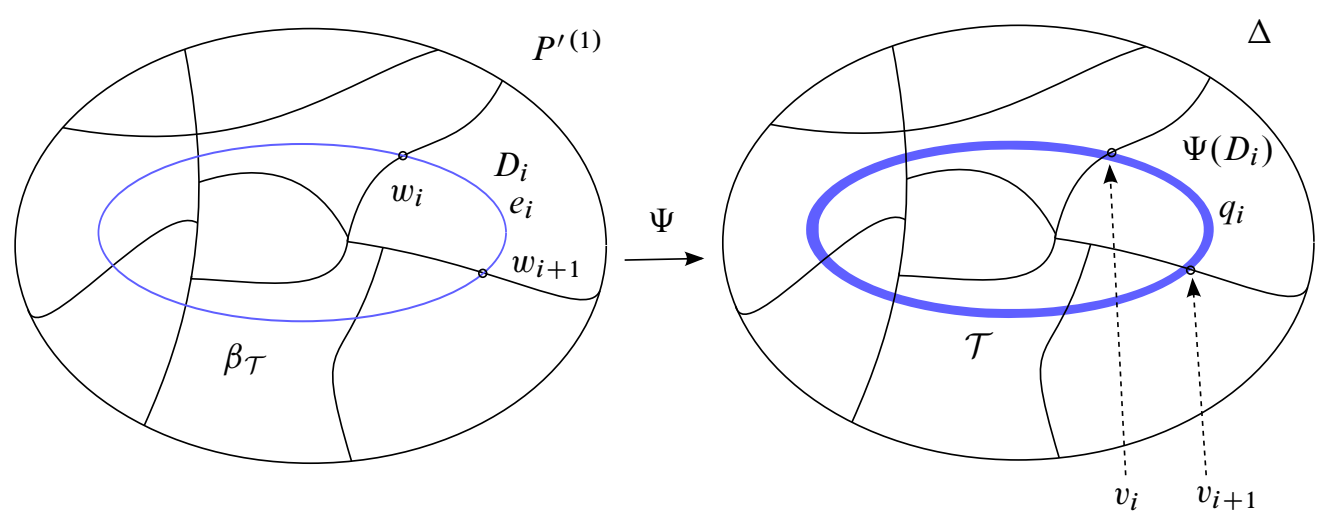

Figure 3: Constructing $P^{\prime}$

We can define $\left.\Psi^{\prime}\right|_{P^{(1)}}=\Psi$ and map $e_{i}$ to $q_{i}$. By Lemma 4.1, we may also assume that $\Psi^{\prime}\left(e_{i}\right)$ has freely reduced label. We can extend $\Psi^{\prime}$ to the 2-cells of $P^{\prime}$ in the natural way. Then $\Psi^{\prime}: P^{\prime(2)} \rightarrow \Delta$ is a partition of $\Delta$ that satisfies the first and third conditions of the claim.

The geodesic condition on crossing vertices guarantees that the mesh does not increase as we add the vertices $v_{i}$ and the edges $e_{i}$.

Claim 4 gives us that $\Psi\left(\beta_{\mathcal{T}}\right)$ bounds a subdiagram of $\Delta$ with freely trivial boundary label and $\beta_{\mathcal{T}}$ bounds a subcomplex of $P^{\prime(1)}$. There exists a simplicial tree $L_{\mathcal{T}}$ labeled by $a$-letters and a map $\Upsilon: \beta_{\mathcal{T}} \rightarrow L_{\mathcal{T}}$ such that $\left.\theta \circ \Psi^{\prime}\right|_{\beta_{\mathcal{T}}}=\theta^{\prime} \circ \Upsilon$, where $\theta^{\prime}$ is a label-preserving map from $L_{\mathcal{T}}$ into $\Gamma(G, S) . L_{\mathcal{T}}$ is constructed by choosing a free reduction of $\mathbf{L a b}\left(\Psi^{\prime}\left(\beta_{\mathcal{T}}\right)\right)$.

We can replace the subdiagram in $\Delta$ bounded by $\Psi\left(\beta_{\mathcal{T}}\right)$ with $L_{\mathcal{T}}$. This creates a pairing of $\Delta$-edges in $\Delta$. What we want to be able to do is mirror this identification of edges on $\beta_{\mathcal{T}}$. The problem is that this identification can pair proper segments of edges in $\beta_{\mathcal{T}}$. To correct this we will need to add new vertices to $P^{\prime}$ to insure that this identification respects $\Psi^{\prime}$-edges. In general, this will cause the mesh to increase since edges of $\beta_{\mathcal{T}}$ do not map to geodesics. So we will subdivide pieces to get a useful bound on our new mesh. This is where the bound $B$ on the diameter of each piece comes into play.

We will say that a subpath of $\beta_{\mathcal{T}}$ is an $L_{\mathcal{T}}$-segment if all vertices of the edge path except possibly the initial and terminal vertices have degree 2 in $\Upsilon\left(\beta_{\mathcal{T}}\right)$.

Claim 5 There exists a refinement $P^{\prime \prime}$ of $P^{\prime}$ and a partition $\Psi^{\prime \prime}: P^{\prime \prime}(2) \rightarrow \Delta$ with $\Psi^{\prime \prime}(x)=\Psi^{\prime}(x)$ for all $x \in P^{\prime \prime(2)}=P^{\prime(2)}$ such that: 
(i) The number of pieces of $\Psi^{\prime \prime}$ is no more than $9 F^{2}+4 F$.

(ii) $\beta_{\mathcal{T}}$ is subdivided into at most $9 F^{2}$ edges and each edge is an $L_{\mathcal{T}}$-segment.

(iii) $\operatorname{rmesh}_{Z}\left(\Psi^{\prime \prime}\right) \leq \max \{3(B+K)$, mesh $(\Psi)\}$, where $Z$ is the set of pieces of $P^{\prime \prime}$ that are not interior to $\beta_{\mathcal{T}}$.

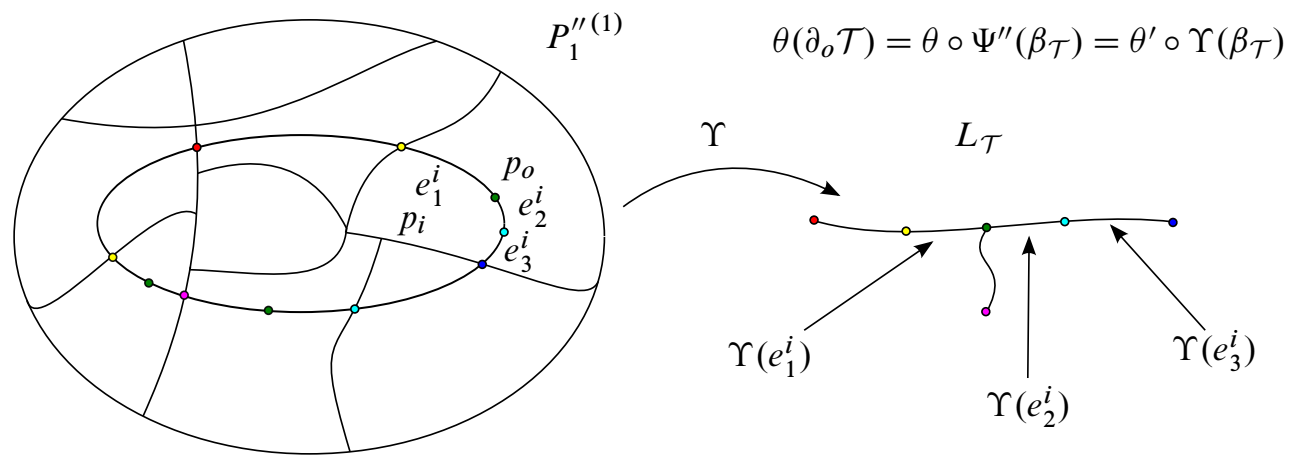

Figure 4: $P_{1}^{\prime \prime(1)}$ and $L_{\mathcal{T}}$

Proof of Claim $5 \Upsilon$ must map each $e_{i}$ injectively into $L_{\mathcal{T}}$, since $\mathbf{L a b}\left(\Psi^{\prime}\left(e_{i}\right)\right)$ is freely reduced. Thus a vertex of $L_{\mathcal{T}}$ with degree 1 must be the image of a vertex of $e_{i}$ for some $i$ and $\Upsilon\left(\beta_{\mathcal{T}}\right)$ has at most $3 F$ vertices of degree 1. Then Lemma 3.23 implies that it has at most $3 F$ vertices of degree greater than 2 . For each $i$, we can add new vertices to $e_{i}$ that are the unique $\Upsilon$-preimage of vertices of $L_{\mathcal{T}}$ with degree greater than 2 or the unique $\Upsilon$-preimage of a point of $\Upsilon(W)$ (see Figure 4). Doing this subdivides $e_{i}$ into at most $3 F$ edges, which we will label by $e_{j}^{i}$ with their ordering induced by $e_{i}$. This divides $\beta_{\mathcal{T}}$ into at most $9 F^{2}$ edges.

Let $P_{1}^{\prime \prime}$ be the cellular decomposition obtained by adding $\left\{e_{i}^{j}\right\}$ to $P^{\prime}$. Notice that $P_{1}^{\prime \prime}$ is not a partition of $A$ since it has vertices of degree 2 .

In $P^{\prime}$ there existed exactly two pieces that share $e_{i}$ as a common edge: $p_{i}$, which is contained in the bounded component of $\mathbb{R}^{2} \backslash \beta_{\mathcal{T}}$, and $p_{o}$, which is contained in the unbounded component (see Figure 4).

We will now subdivide the piece $p_{o}$ to obtain pieces with bounded mesh (see Figure 5). Let $f_{j}^{i}$ be an arc in $p_{o}$ from the initial vertex of $e_{1}^{i}$ to the terminal vertex of $e_{j}^{i}$ for all $j>1$. We also will require that the new edges have disjoint interiors contained in $p_{o}$. This subdivides $p_{o}$ into at most $3 F+1$ pieces, ie, we add $3 F$ pieces to our count. Repeating this process for each $i$ gives us a partition $P^{\prime \prime}$ of $A$. 

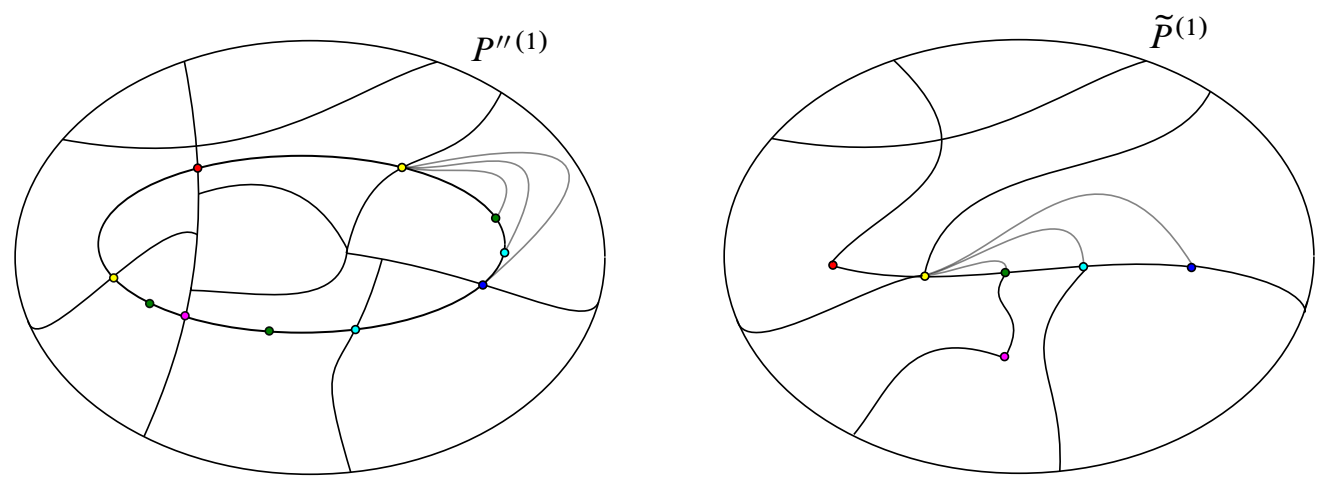

Figure 5: Constructing $\widetilde{P}$

We must now explain how to map these edges into $\Delta$. Each new edge connects points with image on the $\Psi\left(\beta_{\mathcal{T}}\right)$. Thus we can send each edge to the reduced subpath of $\Psi\left(\beta_{\mathcal{T}}\right)$ connecting the images of their vertices and map the 2-cells in the natural way. Let $\Psi^{\prime \prime}: P^{\prime \prime(2)} \rightarrow \Delta$ be this new partition.

The distance between $e_{j}^{i}$ and $e_{j^{\prime}}^{i}$ is at most $B+2 K$ for all $j$ and $j^{\prime}$. This implies that the requirement on the mesh is then satisfied.

We can replace the subdiagram of $\Delta$ bounded by $\Psi^{\prime}\left(\beta_{\mathcal{T}}\right)$ with $L_{\mathcal{T}}$, creating a new van Kampen diagram $\Delta^{\prime}$. This also induces a paring of edges on $\beta_{\mathcal{T}}$ such that after removing the disc bounded by $\beta_{\mathcal{T}}$ and identifying edges of $\beta_{\mathcal{T}}$ according to this pairing, we obtain a new partition $\widetilde{P}$ of the quotient space $A^{\prime}$. If $m(\mathcal{T})$ separates the boundary components of $A$, then $A^{\prime}$ is a planar disc. If $m(\mathcal{T})$ does not separate the boundary components of $A$, then $A^{\prime}$ is an annulus. Then $\Psi^{\prime \prime}$ induces a map $\widetilde{\Psi}: \widetilde{P}^{(2)} \rightarrow \Delta^{\prime}$ with the desired properties; see Figure 5.

Definition 4.11 Recall that $G$ has a presentation

$$
\left.\left\langle A \cup\left\{t_{i}\right\}\right|\left\{u_{i, s}^{t_{i}}=v_{i, s}\right\}, \quad \text { for } i=1, \ldots, k \text { and } s=1, \ldots, j_{i}\right\rangle,
$$

where $U_{i}=\left\langle u_{i, 1}, \ldots, u_{i, j_{i}}\right\rangle, V_{i}=\left\langle v_{i, 1}, \ldots, v_{i, j_{i}}\right\rangle$ are free subgroups with free generating sets $\left\{u_{i, j}\right\},\left\{v_{i, j}\right\}$ respectively and $t_{i}$ are stable letters.

Let $X_{i}$ be the midpoints of the set of edges $\left\{\left(g, t_{i}\right) \mid g \in U_{i}\right\}$ in $\Gamma(G, S)$.

By Britton's Lemma, $g X_{i}$ separates $\Gamma(G, S)$ for every $g \in G$. Let $x_{1}, x_{2}$ be two points in $X_{i}$ such that $x_{2}={ }_{G} x_{1} u_{i, j}$. Then in $\Gamma^{2}(G, S)$ we can find an arc joining $x_{1}$ to $x_{2}$ that intersects $\Gamma(G, S)$ only at $x_{1}$ and $x_{2}$. Let $T_{i}$ be the subset of $\Gamma^{2}(G, S)$ obtained by connecting all such points of $X_{i}$ by arcs that intersect $\Gamma(G, S)$ only at 
their endpoints. Since $U_{i}$ is free, $T_{i}$ is a tree. Then $T_{i}$ separates $\Gamma^{2}(G, S)$ and will be called the median tree for $X_{i}$. Notice that $X_{i}, T_{i}$ are not cellular subset of $\Gamma(G, S)$ or $\Gamma^{2}(G, S)$, even thought they do have a natural cellular structure.

Let $Z, Z^{\prime}$ be subsets of $\Gamma(G, S)$. We will say that $Z, Z^{\prime}$ are $t$-separated if there exists $g \in G$ and $i$ such that $Z, Z^{\prime}$ are in distinct components of $\Gamma(G, S) \backslash g X_{i}$. This is equivalent to saying that as subsets of $\Gamma^{2}(G, S) ; Z, Z^{\prime}$ are in distinct components of $\Gamma^{2}(G, S) \backslash g T_{i}$.

Remark 4.12 Notice that $t$-separated does not imply $U_{i}$-separated or $V_{i}$-separated. Let $Z$ the set of vertices of $\Gamma(G, S)$ that have a label without pinches which begins with the letter $t_{1}$. Let $Z^{\prime}$ be the remainder of the vertices of $\Gamma(G, S)$. Then $Z, Z^{\prime}$ are in distinct components of $\Gamma(G, S) \backslash X_{1}$. Since $Z \cup Z^{\prime}$ contains all the vertices of $G$, they cannot be $U_{i}$-separated or $V_{i}$ separated for any $i$. The point is that $X_{i}$ separates by removing midpoints of edges and $g V_{i}$ or $g U_{i}$ separates by removing vertices.

Lemma 4.13 Suppose $U_{i}$ is proper, $V_{i}$ is proper, or the number of stable letter in $S$ is greater than 1. Let $\gamma$ be a loop in $\Gamma(G, S)$ and $N>\operatorname{diam}(\gamma)$. Then there exist elements $\left\{g_{1}, \ldots, g_{N}\right\}$ in $G$ such that $g_{i} \cdot \gamma, g_{j} \cdot \gamma$ are $t$-separated and $\left|g_{i} g_{j}^{-1}\right| \geq 2 N$ for all $i \neq j$, and $\left|g_{i}\right| \leq 4 N$.

Proof If $U_{i}$ or $V_{i}$ is proper, then $\left\{g_{j}\right\}$ can be constructed as in Lemma 3.14. If $S$ has at least two stable letters, then let $g_{i}=t_{1}^{N} t_{2}^{i} t_{1}^{-N}$. In any of the three cases, the proof of Lemma 3.14 also shows that the loops $\left\{g_{j} \cdot \gamma\right\}$ are pairwise $t$-separated.

Lemma 4.14 Suppose that $\theta: \Delta^{(2)} \rightarrow \Gamma^{2}(G, S)$ is the canonical label-preserving cellular map from a van Kampen diagram $\Delta$ over $\langle S \mid R\rangle$ to the Cayley complex. Then $\theta^{-1}\left(g T_{i}\right)$ is a set of medians of $t_{i}$-bands in $\Delta$.

Proof The only cells in $\Gamma^{2}(G, S)$ intersecting $g T_{i}$ are those corresponding to relations of the form $u_{i, j}^{t}=v_{i, j}$. The preimage of each edge of $g T_{i}$ is a median of such a cell in $\Delta$.

Lemma 4.15 Suppose that $\Delta_{A}$ is an annular diagram such that the components of $\theta\left(\partial \Delta_{A}\right)$ are $t$-separated. Then there exists a $t$-annulus in $\Delta_{A}$ that separates the boundary components of $\Delta_{A}$.

Proof Since the components of $\theta\left(\partial \Delta_{A}\right)$ are $t$-separated, there exist $g \in G$ and $i$ such that they are in distinct components of $\Gamma^{2}(G, S) \backslash g T_{i}$. Then $\theta^{-1}\left(g T_{i}\right)$ separates the components of $\partial \Delta_{A}$ and the result follows from Lemma 4.14 . 
Theorem 4.16 Let $G$ be a multiple HNN of a free group with free associated subgroups. Then either all asymptotic cones of $G$ are simply connected or $G$ has an asymptotic cone with uncountable fundamental group.

Proof If $G$ has only one stable letter and both associated subgroups are not proper, then $G$ has a quadratic Dehn function (see Bridson and Groves [1]) and every asymptotic cone of $G$ is simply connected.

If there exists an asymptotic cone of $G$ that is not simply connected, then there exists a sequence of loops $\gamma_{n}$ in $\Gamma(G, S)$ such that $P\left(\gamma_{n},\left|\gamma_{n}\right| / 2\right) \geq n$ for all n. Let $d_{n}=\left|\gamma_{n}\right|$. Then $d_{n}$ diverges $\omega$-almost surely and $\gamma(t)=\left(\gamma_{n}(t)\right)$ is a loop that has no finite partition in $\operatorname{Con}^{\omega}(G, d)$.

Using Lemma 4.13, we can choose a set $S_{n}=\left\{g_{n, 1}, \ldots, g_{n, k_{n}}\right\}$ of elements of $G$ such that

(a) if $i \neq j$, then $g_{n, i} \cdot \gamma_{n}$ and $g_{n, j} \cdot \gamma_{n}$ are $t$-separated and

(b) for all $i, 2 \operatorname{diam}(\gamma) d_{n} \leq\left|g_{n, i}\right| \leq 4 \operatorname{diam}(\gamma) d_{n}$.

Claim Let $g=\left(g_{n}\right), h=\left(h_{n}\right)$ be distinct elements in $\prod^{\omega} S_{n}$. Then $g \cdot \gamma$ is a well-defined loop $\operatorname{Con}^{\omega}(G, d)$ and $g \cdot \gamma$ is not homotopic to $h \cdot \gamma$.

The first assertion follows from the fact that $g_{n}$ grows big $O$ of the scaling sequence.

Suppose that $g \cdot \gamma$ is homotopic to $h \cdot \gamma$. Then we have a homotopy $h: A \rightarrow \operatorname{Con}^{\omega}(G, d)$ between the two loops where $A$ is a planar annulus. Let $P$ be a partition of $A$, where each piece is a triangle such that $\operatorname{diam}(h(D)) \leq 1 /(84 L)$ for each piece $D$ of $P$. Then we can chose partitions $\Pi_{n}: P^{(0)} \rightarrow \Gamma(G, S)$ such that $\left(\Pi_{n}(x)\right)=h(x)$ for all $x \in P^{(0)}$. As in Remark 4.5, $\Pi_{n}$ induces a $t$-shortest partition $\Psi_{n}: P^{(2)} \rightarrow \Delta_{n}^{\prime}$ where $\Delta_{n}^{\prime}$ is an annular van Kampen diagram where both boundary paths are labeled by $\mathbf{L a b}\left(\gamma_{n}\right)$. Then

$$
\operatorname{mesh}\left(\Psi_{n}\right) \leq \frac{\left|\gamma_{n}\right|}{60 L}+o\left(\left|\gamma_{n}\right|\right)<\frac{\left|\gamma_{n}\right|}{30 L}
$$

$\omega$-almost surely. Lemma 4.4 implies that the $\operatorname{diam}(\theta \circ \Psi(D)) \leq 5 L$ mesh $(\Psi)<\left|\gamma_{n}\right| / 6$ $\omega$-almost surely.

Since $g \neq h, g_{n} \neq h_{n} \omega$-almost surely and the loops $g_{n} \cdot \gamma_{n}$ and $h_{n} \cdot \gamma_{n}$ are $t$ separated $\omega$-almost surely. Lemma 4.15 implies that there exists a $t$-annulus in $\Delta_{n}$ that separates the two boundary components of $\Delta_{n} \omega$-almost surely. Lemma 4.10 implies we can remove this $t$-annulus to obtain a partition $\tilde{\Psi}_{n}$ of a circular diagram $\Delta_{n}^{\prime}$ with $\mathbf{L a b}\left(\partial \Delta_{n}^{\prime}\right)=\mathbf{L a b}\left(\gamma_{n}\right) \omega$-almost surely. Notice that mesh $\left(\widetilde{\Psi}_{n}\right)<3\left(\left(\left|\gamma_{n}\right| / 6\right)+K\right)$ and has at most $9 F^{2}+4 F$, where $F$ is the number of pieces of $P$. This then contradicts our choice of $\gamma_{n}$. 


\section{References}

[1] M R Bridson, D Groves, The quadratic isoperimetric inequality for mapping tori of free group automorphisms, Mem. Amer. Math. Soc. 955 (2010) MR2590896

[2] M R Bridson, A Haefliger, Metric spaces of non-positive curvature, Grundl. Math. Wissen. 319, Springer, Berlin (1999) MR1744486

[3] J Burillo, Dimension and fundamental groups of asymptotic cones, J. London Math. Soc. 59 (1999) 557-572 MR1709665

[4] J W Cannon, G R Conner, The combinatorial structure of the Hawaiian earring group, Topology Appl. 106 (2000) 225-271 MR1775709

[5] J W Cannon, G R Conner, On the fundamental groups of one-dimensional spaces, Topology Appl. 153 (2006) 2648-2672 MR2243740

[6] D J Collins, J Huebschmann, Spherical diagrams and identities among relations, Math. Ann. 261 (1982) 155-183 MR675732

[7] G Conner, C Kent, Local topological properties of asymptotic cones of groups arXiv: 1210.4411

[8] Y Cornulier, R Tessera, Dehn function and asymptotic cones of Abels' group, J. Topol. $6(2013)$

[9] N DeJarnette, P Hajlasz, A Lukyanenko, J Tyson, On the lack of density of Lipschitz mappings in Sobolev spaces with Heisenberg target arXiv:1109.4641

[10] C Druţu, S Mozes, M V Sapir, Divergence in lattices in semisimple Lie groups and graphs of groups, Trans. Amer. Math. Soc. 362 (2010) 2451-2505 MR2584607

[11] C Druţu, M V Sapir, Tree-graded spaces and asymptotic cones of groups, Topology 44 (2005) 959-1058 MR2153979

[12] D B A Epstein, J W Cannon, D F Holt, S V F Levy, M S Paterson, W P Thurston, Word processing in groups, Jones and Bartlett, Boston, MA (1992) MR1161694

[13] A Erschler, D V Osin, Fundamental groups of asymptotic cones, Topology 44 (2005) 827-843 MR2136537

[14] R Geoghegan, The shape of a group - connections between shape theory and the homology of groups, from: "Geometric and algebraic topology", (H Toruńczyk, S Jackowski, S Spież, editors), Banach Center Publ. 18 (1986) 271-280 MR925870

[15] H B Griffiths, The fundamental group of two spaces with a common point: A correction, Quart. J. Math. Oxford Ser. 6 (1955) 154-155 MR0070167

[16] M Gromov, Asymptotic invariants of infinite groups, from: "Geometric group theory, Vol. 2”, (G A Niblo, M A Roller, editors), London Math. Soc. Lecture Note Ser. 182, Cambridge Univ. Press (1993) 1-295 MR1253544

[17] V S Guba, The Dehn function of Richard Thompson's group $F$ is quadratic, Invent. Math. 163 (2006) 313-342 MR2207020 
[18] W Hurewicz, H Wallman, Dimension theory, Princeton Mathematical Series 4, Princeton Univ. Press (1941) MR0006493

[19] L Kramer, S Shelah, K Tent, S Thomas, Asymptotic cones of finitely presented groups, Adv. Math. 193 (2005) 142-173 MR2132762

[20] L Mosher, Mapping class groups are automatic, Ann. of Math. 142 (1995) 303-384 MR1343324

[21] J R Munkres, Topology: A first course, Prentice-Hall, New Jersey (1975) MR0464128

[22] A Y Ol'shanskii, Geometry of defining relations in groups, Mathematics and its Applications (Soviet Series) 70, Kluwer, Dordrecht (1991) MR1191619

[23] A Y Ol'shanskii, D V Osin, M V Sapir, Lacunary hyperbolic groups, Geom. Topol. 13 (2009) 2051-2140 MR2507115

[24] A Y Ol'shanskii, M V Sapir, Groups with non-simply connected asymptotic cones, from: "Topological and asymptotic aspects of group theory", Contemp. Math. 394, Amer. Math. Soc. (2006) 203-208 MR2216717

[25] A Y Ol'shanskii, M V Sapir, Groups with small Dehn functions and bipartite chord diagrams, Geom. Funct. Anal. 16 (2006) 1324-1376 MR2276542

[26] A Y Ol'shanskii, M V Sapir, A finitely presented group with two non-homeomorphic asymptotic cones, Internat. J. Algebra Comput. 17 (2007) 421-426 MR2310154

[27] D V Osin, M V Sapir, Universal tree-graded spaces and asymptotic cones, Internat. J. Algebra Comput. 21 (2011) 793-824 MR2827204

[28] P Pansu, Croissance des boules et des géodésiques fermées dans les nilvariétés, Ergodic Theory Dynam. Systems 3 (1983) 415-445 MR741395

[29] P Papasoglu, On the asymptotic cone of groups satisfying a quadratic isoperimetric inequality, J. Differential Geom. 44 (1996) 789-806 MR1438192

[30] F Point, Groups of polynomial growth and their associated metric spaces, J. Algebra 175 (1995) 105-121 MR1338969

[31] M V Sapir, J-C Birget, E Rips, Isoperimetric and isodiametric functions of groups, Ann. of Math. 156 (2002) 345-466 MR1933723

[32] A Sisto, Tree-graded asymptotic cones, Groups Geom. Dyn. 7 (2013) 697-735 MR3095715

[33] R Young, The Dehn function of $\operatorname{SL}(n ; \mathbb{Z})$, Ann. of Math. 177 (2013) 969-1027 MR3034292

Mathematics Department, University of Toronto

Room 6290, 40 St. George Street, Toronto, ON M5S 2E4, Canada

curt.kent@utoronto.ca

http: //www.math.toronto.edu/cms/kent-curt/

Received: 16 October 2012 Revised: 9 January 2013 